\title{
TRANSFORMATION OF POLYNOMIALS ORTHOGONAL ON THE UNIT CIRCLE
}

\section{Franz Peherstorfer and Robert Steinbauer}

\begin{abstract}
Let $\left\{a_{n}\right\}$ be an arbitrary sequence of real numbers with $\left|a_{n}\right|<1$ for $n \in \mathbb{N}_{0}$, and let $b_{1}, \ldots, b_{N-1}, b_{N-j}=-\overline{b_{j}}$ be an arbitrary symmetric sequence of $N-1$ complex numbers with $\left|b_{j}\right|<1$. In this paper, we give a full and explicit description of the orthogonal polynomials, the measures, and the Carathéodory functions which are associated with reflection coefficients of the form $\left\{b_{1}, \ldots, b_{N-1}, a_{0}, b_{1}, \ldots, b_{N-1}, a_{1}, \ldots\right\}$. More precisely, we show that the orthogonal polynomials generated by such a sequence of reflection coefficients can be obtained by a transformation of polynomials orthogonal on the unit circle. Further, they are orthogonal with respect to a measure of the form $(w(\varphi) / \sqrt{\rho(\varphi)}$ $\left.\vartheta^{\prime}(\varphi)\right) d \sigma_{0}(\vartheta(\varphi))$ where $\sigma_{0}$ is the orthogonality measure associated with $\left\{a_{n}\right\}$ and where the trigonometric polynomials $w, \rho$ and the function $\vartheta$ can be constructed explicitly with the help of the $b_{j}$ 's. In particular, $\vartheta$ is a function which maps $N$ subintervals of $[0,2 \pi]$ onto the whole interval $[0,2 \pi]$. On the other hand, it is demonstrated that polynomials, which are orthogonal with respect to a measure $\left(w(\varphi) / \sqrt{\rho(\varphi)} \vartheta^{\prime}(\varphi)\right) d \sigma_{0}(\vartheta(\varphi))$ where $\sigma_{0}$ is an arbitrary symmetric measure on $[0,2 \pi]$ and $\vartheta$ is a function of the type just given, have reflection coefficients of the above form.
\end{abstract}

\section{Introduction and notation}

Let $\sigma_{0}(\varphi)$ be a positive Borel measure on the interval $[0,2 \pi]$ with an infinite support. Then for every $n \in \mathbb{N}_{0}$, there exists a uniquely determined monic orthogonal polynomial $P_{n}\left(z, \sigma_{0}\right)=z^{n}+\cdots$ of degree $n$ which satisfies

$$
\int_{0}^{2 \pi} e^{-i j \varphi} P_{n}\left(e^{i \varphi}, \sigma_{0}\right) d \sigma_{0}(\varphi)=0 \quad \text { for } j=0, \ldots, n-1 .
$$

It is well known that these monic orthogonal polynomials can be generated by the recurrence formula

$$
P_{n+1}\left(z, \sigma_{0}\right)=z P_{n}\left(z, \sigma_{0}\right)-\overline{a_{n}\left(\sigma_{0}\right)} P_{n}^{*}\left(z, \sigma_{0}\right), \quad n \in \mathbb{N}_{0}
$$

where $P_{n}^{*}\left(z, \sigma_{0}\right):=z^{n} \overline{P_{n}\left(1 / \bar{z}, \sigma_{0}\right)}$ is the reversed polynomial of $P_{n}\left(z, \sigma_{0}\right)$. Here, the complex numbers $a_{n}\left(\sigma_{0}\right)=-\overline{P_{n+1}\left(0, \sigma_{0}\right)}$ satisfy

$$
\left|a_{n}\left(\sigma_{0}\right)\right|<1, \quad n \in \mathbb{N}_{0},
$$

and are called reflection coefficients or Schur parameters.

Received March 4, 1997, revised October 27, 1997.

1991 Mathematics Subject Classification: $42 \mathrm{C} 05$.

Key words and phrases: orthogonal polynomials, unit circle, composition, reflection coefficients. 
There is a one-to-one correlation between the measure $\sigma_{0}$ and the Carathéodory function (abbreviated in the following by C-function) $F\left(z, \sigma_{0}\right)$, which is defined by

$$
F\left(z, \sigma_{0}\right):=\int_{0}^{2 \pi} \frac{e^{i \varphi}+z}{e^{i \varphi}-z} d \sigma_{0}(\varphi), \quad|z|<1
$$

$F\left(z, \sigma_{0}\right)$ is analytic on $|z|<1$ and pseudopositive there, i.e., $\operatorname{Re} F\left(z, \sigma_{0}\right)>0$ for $|z|<1$. The distribution function $\sigma_{0}$ can be reconstructed from $F\left(z, \sigma_{0}\right)$ by means of the inversion formula

$$
\frac{\sigma_{0}(\varphi+0)+\sigma_{0}(\varphi-0)}{2}=\text { constant }+\lim _{s \rightarrow 1^{-}} \frac{1}{2 \pi} \int_{0}^{\varphi} \operatorname{Re} F\left(s e^{i \psi}, \sigma_{0}\right) d \psi
$$

Next, let us give the definition of the (not necessarily monic) polynomials of the second kind $\Omega_{n}\left(z, \sigma_{0}\right)$ with respect to $\sigma_{0}$ :

$$
\Omega_{n}\left(z, \sigma_{0}\right):= \begin{cases}\int_{0}^{2 \pi} \frac{e^{i \varphi}+z}{e^{i \varphi}-z}\left(P_{n}\left(e^{i \varphi}, \sigma_{0}\right)-P_{n}\left(z, \sigma_{0}\right)\right) d \sigma_{0}(\varphi) & \text { if } n \geq 1, \\ F\left(0, \sigma_{0}\right) & \text { if } n=0 .\end{cases}
$$

It is not difficult to see that all the $\Omega_{n}$ 's are of exact degree $n$ with leading coefficient $F\left(0, \sigma_{0}\right)$. Furthermore, the polynomials of the second kind satisfy the recurrence relation similar to (1.1), namely,

$$
\Omega_{n+1}\left(z, \sigma_{0}\right)=z \Omega_{n}\left(z, \sigma_{0}\right)+\overline{a_{n}\left(\sigma_{0}\right)} \Omega_{n}^{*}\left(z, \sigma_{0}\right), \quad n \in \mathbb{N}_{0} .
$$

Another known and useful relation between the orthogonal polynomials and the polynomials of the second kind is

$$
P_{n}^{*}\left(z, \sigma_{0}\right) \Omega_{n}\left(z, \sigma_{0}\right)+P_{n}\left(z, \sigma_{0}\right) \Omega_{n}^{*}\left(z, \sigma_{0}\right)=2 F\left(0, \sigma_{0}\right) d_{n}\left(\sigma_{0}\right) z^{n}
$$

(see e.g., [8, formula (5.6)]), where $d_{n}\left(\sigma_{0}\right):=\prod_{j=0}^{n-1}\left(1-\left|a_{j}\left(\sigma_{0}\right)\right|^{2}\right)$ if $n>0$ and $d_{0}\left(\sigma_{0}\right):=1$.

With the help of the C-function and the polynomials of the second kind, the orthogonal polynomials can be characterized in the following way.

Theorem 1 (Peherstorfer and Steinbauer [17]). Let $\sigma_{0}$ be a distribution function. Then a polynomial $A$ of degree $n$ is orthogonal with respect to $\sigma_{0}$, i.e., $A=c P_{n}\left(\cdot, \sigma_{0}\right)$, $c \in \mathbb{C} \backslash\{0\}$, if and only if there exists a polynomial $B$ of degree $n$ such that

$$
\begin{aligned}
A(z) F\left(z, \sigma_{0}\right)+B(z) & =\mathcal{O}\left(z^{n}\right), \\
A^{*}(z) F\left(z, \sigma_{0}\right)-B^{*}(z) & =\mathcal{O}\left(z^{n+1}\right) .
\end{aligned}
$$

In such a case, $B$ is the polynomial of the second kind with respect to $\sigma_{0}$, i.e., $B=$ $c \Omega_{n}\left(\cdot, \sigma_{0}\right)$.

It is well known (compare the above considerations) that to each sequence of reflection coefficients $\left\{a_{n}\left(\sigma_{0}\right)\right\}$, there corresponds an orthogonality measure $\sigma_{0}$ and to the measure $\sigma_{0}$, a C-function $F\left(z, \sigma_{0}\right)$ in a unique way. Thus, we have the following correspondence :

$$
\left\{a_{n}\left(\sigma_{0}\right)\right\} \longleftrightarrow \sigma_{0}(\varphi) \longleftrightarrow F\left(z, \sigma_{0}\right) .
$$

One of the main problems now is to find the corresponding measure $\sigma_{0}$ (respectively, the C-function $\left.F\left(z, \sigma_{0}\right)\right)$ for a given sequence of reflection coefficients $\left\{a_{n}\left(\sigma_{0}\right)\right\}$ and 
conversely. Cases where solutions can be found explicitly (i.e., the orthogonality measure as well as the reflection coefficients can be given explicitly), are very rare: only the Jacobi weight functions $(1-\cos \varphi)^{\alpha}(1+\cos \varphi)^{\beta}|\sin \varphi|$ are studied (see e.g., [15, p.234]) and - from the point of the reflection coefficients - the case of periodic reflection coefficients (e.g., see $[7,19])$. If one knows the solution for the sequence $\left\{a_{n}\right\}$, then also one can handle a finite perturbation of the reflection coefficients [16] and the case when the reflection coefficients are of the form $\left\{0, \ldots, 0, a_{0}, 0, \ldots, 0, a_{1}, \ldots\right\}$ where there always appear $N$ successive zeros. For instance, by $[1,11,14,17]$, we have the following correspondence:

$$
\{\underbrace{0, \ldots, 0}_{N \text { times }}, a_{0}\left(\sigma_{0}\right), \underbrace{0, \ldots, 0}_{N \text { times }}, a_{1}\left(\sigma_{0}\right), 0, \ldots\} \quad \longleftrightarrow \quad \sigma_{0}(N \varphi) \longleftrightarrow F\left(z^{N}, \sigma_{0}\right),
$$

assuming (1.5) holds. Moreover, the orthogonal polynomials with respect to $\sigma_{0}(N \varphi)$ where $N \varphi$ is taken modolo $2 \pi$, are explicitly given by $z^{j} P_{n}\left(z^{N}, \sigma_{0}\right), j=0, \ldots, N-1$. Hence, it is natural to ask (see [21, pp. 142-145] and [11]), whether such a correspondence also can be given for reflection coefficients of the form

$$
\left\{b_{1}, \ldots, b_{N-1}, a_{0}\left(\sigma_{0}\right), b_{1}, \ldots, b_{N-1}, a_{1}\left(\sigma_{0}\right), \ldots\right\}
$$

where the $b_{j}, j=1, \ldots, N-1$ are complex numbers with $\left|b_{j}\right|<1$. For many of the specialists in this field, a correspondence with a C-function of the form $F\left(T_{N}(z), \sigma_{0}\right)$, $T_{N}$ a polynomial, was expected. But this does not hold in general. It will turn out that it is more natural to study a functional transformation which maps $N$ arcs of the unit circle onto the whole unit circle, instead of a polynomial transformation. Under the additional assumptions that the starting-reflection coefficients $a_{n}\left(\sigma_{0}\right)$ are real and that the inserted reflection coefficients $b_{j}$ are symmetric in the sense that

$$
\overline{b_{j}}=-b_{N-j}, \quad j=1, \ldots, N-1,
$$

we shall demonstrate a correspondence of the following form:

$$
\begin{aligned}
\{\underbrace{b_{1}, \ldots, b_{N-1}}_{N \text { times }}, a_{0}\left(\sigma_{0}\right), \underbrace{b_{1}, \ldots, b_{N-1}}_{N \text { times }}, a_{1}\left(\sigma_{0}\right), b_{1}, \ldots\} & \longleftrightarrow \\
\frac{W\left(e^{i \varphi}\right)}{\sqrt{R\left(e^{i \varphi}\right)} \vartheta^{\prime}(\varphi)} d \sigma_{0}(\vartheta(\varphi)) & \longleftrightarrow \frac{W(z)}{\sqrt{R(z)}} F\left(\frac{\mathcal{T}(z)-\sqrt{R(z)}}{\mathcal{T}(z)+\sqrt{R(z)}}, \sigma_{0}\right) .
\end{aligned}
$$

Here the polynomials $R, \mathcal{T}$, and $W$ only depend on the values of $b_{1}, \ldots, b_{N-1}$, and the function $\vartheta$ is given by

$$
\vartheta(\varphi)=\operatorname{Arg}\left\{\frac{\mathcal{T}(z)-\sqrt{R(z)}}{\mathcal{T}(z)+\sqrt{R(z)}}\right\}
$$

for detailed description, see the next section.

This paper is organized as follows: In Section 2, we will state some additional preliminary notations and definitions, and in Section 3, we give a full explicit description of the correspondence in (1.7). We also will show how the resulting orthogonal polynomials are related to the original orthogonal polynomials $P_{n}\left(z, \sigma_{0}\right)$. Making use of the well-known relationship between orthogonal polynomials on the unit circle and on the real line, our results reprove some known results of Geronimo and Van Assche [9] but also give some new insights to the reflection coefficients of orthogonal polynomials on the real line; see Section 4. In Section 5 , we give the proofs of our results. 


\section{Preliminaries}

In this section, and for the rest of the paper, we suppose that $\sigma_{0}$ is a symmetric probability measure, i.e.,

$$
\int_{0}^{2 \pi} d \sigma_{0}(\varphi)=1
$$

and

$$
\begin{gathered}
d \sigma_{0}(\varphi)+d \sigma_{0}(2 \pi-\varphi)=0, \quad \text { or equivalently, } \\
\operatorname{Re} F\left(e^{i \varphi}, \sigma_{0}\right)=\operatorname{Re} F\left(e^{i(2 \pi-\varphi)}, \sigma_{0}\right) \quad \text { a.e. on }[0,2 \pi] .
\end{gathered}
$$

Here, $F\left(e^{i \varphi}, \sigma_{0}\right)$ is considered to be the boundary value $\lim _{s \rightarrow 1^{-}} F\left(s e^{i \varphi}, \sigma_{0}\right)$, which exists a.e. on the unit circle. The symmetry property implies that all the orthogonal polynomials $P_{n}\left(z, \sigma_{0}\right)$ have real coefficients, in particular, the reflection coefficients $a_{n}\left(\sigma_{0}\right)$ are real as well. Furthermore, $F\left(x, \sigma_{0}\right)$ takes real values for $x \in(-1,1)$.

We start our investigations on (1.7) from the point of view of measures, respectively, C-functions. We will introduce a transformation which leads, as it will turn out, exactly to the reflection coefficients of the form given in (1.7) satisfying the properties under consideration. This transformation reproduces the measure $\sigma_{0}$ on several subintervals of $[0,2 \pi]$, similar to the transformation $\varphi \longrightarrow N \varphi \bmod 2 \pi$ from (1.6). In fact, we will arrive at an extension of this function.

For the exact description of our transformation, some auxiliaries are needed: Let $N$ be a positive integer and let $\mathcal{T}(z)=\mathcal{T}^{*}(z)=\alpha z^{N}+\cdots,|\alpha|=1$, be a self-reversed polynomial of degree $N$, whose zeros are all simple and located on the unit circle. Further, let $L>0$ be a real number such that the function $\left|\mathcal{T}\left(e^{i \varphi}\right)\right|-L$ has exactly $2 N$ zeros (counted according to their multiplicity) on $[0,2 \pi)^{1}$.

Finally, we define the polynomial $R$ by

$$
R(z):=\mathcal{T}^{2}(z)-L^{2} z^{N}=\alpha^{2} z^{2 N}+\cdots .
$$

By construction, all the zeros of $R$ lie on the unit circle and are, at most, double.

To the polynomial $\mathcal{T}$ (respectively, $R$ ), we associate the set $E_{N}$, defined by

$$
E_{N}:=\left\{\left|\mathcal{T}\left(e^{i \varphi}\right)\right| \leq L\right\} \quad \bmod 2 \pi=: \bigcup_{j=1}^{N}\left[\varphi_{2 j-1}, \varphi_{2 j}\right] .
$$

Note that the $e^{i \varphi_{j}}$ 's (again counted according to their multiplicity) are exactly the zeros of $R$.

Remark. Let us note that for convenience of the reader that we use a slightly different notation from that in [20]. Here, in contrast to [20], $R$ may have double zeros, i.e., we have

$$
\varphi_{1}<\varphi_{2} \leq \varphi_{3}<\varphi_{4} \leq \varphi_{5}<\cdots<\varphi_{2 N} .
$$

Assuming that exactly $N-l$ of the $\varphi_{j}$ 's coincide, $R$ can be written in the form

$$
R(z)=\tilde{R}(z) \mathcal{U}_{N-l}^{2}(z) \quad \text { and } \quad E_{N}=\tilde{E}_{l}=\bigcup_{\nu=1}^{l}\left[\varphi_{2 j(\nu)-1}, \varphi_{2 j(\nu)}\right]
$$

\footnotetext{
${ }^{1}$ In fact, there exists a $L_{0}>0$ such that all $L \in\left(0, L_{0}\right]$ have the assumed property.
} 
where $\mathcal{U}_{N-l}(z)=\beta z^{N-l}+\cdots$ is a self-reversed polynomial, and the $e^{i \varphi_{j(\nu)}}$ 's are exactly the simple zeros of $R$, i.e., the zeros of $\tilde{R}$. Now $\tilde{R}, \tilde{E}_{l}$, and $\mathcal{U}_{N-l}$ from (2.5) correspond to $R, E_{l}$, and $\mathcal{U}_{N-l}$ from [20].

Now we are ready to define the function $\Theta$, which is essential in what follows, by

$$
\Theta(z):=\frac{\mathcal{T}(z)-\sqrt{R(z)}}{\mathcal{T}(z)+\sqrt{R(z)}} .
$$

Here, the square-root $\sqrt{R}$ is analytic on $\mathbb{C} \backslash \Gamma_{E_{N}}, \Gamma_{E_{N}}:=\left\{e^{i \varphi}: \varphi \in E_{N}\right\}$, and satisfies

$$
\mathcal{T}(0)=\sqrt{R(0)}=\bar{\alpha}
$$

and

$$
\sqrt{R\left(e^{i \varphi}\right)}=\left\{\begin{array}{lll}
(-1)^{j+1} i e^{i(N / 2) \varphi} \sqrt{\left|R\left(e^{i \varphi}\right)\right|}, & \varphi \in\left[\varphi_{2 j-1}, \varphi_{2 j}\right], & j=1, \ldots, N \\
(-1)^{j+1} e^{i(N / 2) \varphi} \sqrt{\left|R\left(e^{i \varphi}\right)\right|}, & \varphi \in\left[\varphi_{2 j}, \varphi_{2 j+1}\right], & j=1, \ldots, N-1 .
\end{array}\right.
$$

The next proposition gives some important properties of the function $\Theta$.

Proposition 1. Let the function $\Theta(z)$ be defined as in (2.6) and suppose that $\mathbb{C} \backslash \Gamma_{E_{N}}$ is connected, i.e., that $\Gamma_{E_{N}}$ is a strict subset of the unit circle. Then the following holds.

(a) $\Theta$ is analytic on $\mathbb{C} \backslash \Gamma_{E_{N}}$ and its only zero is at $z=0$. Further,

$$
\left.\frac{\Theta(z)}{z^{N}}\right|_{z=0}=\left(\frac{L \alpha}{2}\right)^{2}
$$

(b) $\Theta$ maps $\mathbb{C} \backslash \Gamma_{E_{N}}$ onto the interior of the unit disk.

(c) The function

$$
\frac{z^{N / 2}}{\sqrt{\Theta(z)}}=\frac{\mathcal{T}(z)+\sqrt{R(z)}}{L}
$$

maps $\mathbb{C} \backslash \Gamma_{E_{N}}$ onto the exterior of the unit disk.

(d) $\left|\Theta\left(e^{i \varphi}\right)\right|=1$ for $\varphi \in E_{N}$ and $\Theta\left(e^{i \varphi}\right) \in(-1,1)$ for $\varphi \notin E_{N}$.

(e) The mapping $\Theta$ is continuous and bijective on $\left[\varphi_{2 j-1}, \varphi_{2 j}\right), j=1, \ldots, N$, with

$$
\Theta\left(e^{i \varphi_{2 j-1}}\right)=\Theta\left(e^{i \varphi_{2 j}}\right)=1
$$

and

$$
\Theta\left(\Gamma_{\left[\varphi_{2 j-1}, \varphi_{2 j}\right]}\right)=\Gamma_{[0,2 \pi]}=\{|z|=1\}
$$

where we used the notation $\Gamma_{M}:=\left\{e^{i \varphi}: \varphi \in M\right\}$ for $M \subseteq[0,2 \pi]$.

(f) Let the (multivalued) function $Y$ be given by

$$
Y(z):=\sqrt[2 N]{\frac{z^{N}}{\Theta(z)}}=\sqrt[N]{\frac{\mathcal{T}(z)+\sqrt{R(z)}}{L}}
$$

Then $\ln |Y(z)|$ is the Green's function of $\mathbb{C} \backslash \Gamma_{E_{N}}$ with pole at infinity (for the definition of Green's function, e.g., see [24]).

(g) The function

$$
\vartheta(\varphi):=\operatorname{Arg} \Theta\left(e^{i \varphi}\right)
$$


is strictly monotone increasing from 0 to $2 \pi$ on each interval $\left[\varphi_{2 j-1}, \varphi_{2 j}\right]$. Here, the argument Arg $z$ of a complex number $z$ is always considered to be between 0 and $2 \pi$.

(h) The following relations hold:

$$
L \cos \frac{\vartheta(\varphi)}{2}=\tau(\varphi) \quad \text { and } \quad L \sin \frac{\vartheta(\varphi)}{2}=(-1)^{j} \sqrt{|\rho(\varphi)|},
$$

$\varphi \in\left[\dot{\varphi}_{2 j-1}, \varphi_{2 j}\right]$, where the real trigonometric polynomials $\tau(\varphi)$ and $\rho(\varphi)$ are given by

$$
\tau(\varphi):=e^{-i(N / 2) \varphi} \mathcal{T}\left(e^{i \varphi}\right) \quad \text { and } \quad \rho(\varphi):=e^{-i N \varphi} R\left(e^{i \varphi}\right) .
$$

Remark. If $\Gamma_{E_{N}}$ coincides with the unit circle, then $\mathcal{T}(z)=z^{N}+1, R(z)=\left(z^{N}-1\right)^{2}$, and $L=2$. Hence, $\Theta(z)=z^{N}$, and one immediately sees that the parts (a), (d), (e), $(\mathrm{g})$, and (h) of Proposition 1 remain valid for this case.

Given the measure $\sigma_{0}$, respectively, the C-function $F\left(z, \sigma_{0}\right)$, we can construct a new measure $\sigma$ and C-function $F(z, \sigma)$, respectively, via the transformation $\Theta$ in the following way.

Proposition 2. Let $W=-W^{*}$ be an antiself-reversed polynomial of degree $N$ vanishing only at zeros of $R$, which has the same sign as $\sqrt{R\left(e^{i \varphi}\right)}$ on the arcs of $\Gamma_{E_{N}}$ and which is normalized by $|W(0) / \sqrt{R(0)}|=1$. Then the function

$$
F(z, \sigma):=\frac{W(z)}{\sqrt{R(z)}} F\left(\Theta(z), \sigma_{0}\right)-i \operatorname{Im} \gamma
$$

$\gamma:=W(0) / \sqrt{R(0)}$, is a $C$-function where $\sigma$ denotes the associated measure. Furthermore, $\operatorname{supp}(\sigma) \subseteq E_{N}$ and the measure $\sigma$ is given explicitly by

$$
\int_{E_{N}} h(\varphi) d \sigma(\varphi)=\int_{E_{N}} h(\varphi) \frac{W\left(e^{i \varphi}\right)}{\sqrt{R\left(e^{i \varphi}\right)}} \frac{d \sigma_{0}(\vartheta(\varphi))}{\vartheta^{\prime}(\varphi)}
$$

for every continuous function $h$.

Remark. A class of neat looking weight functions obtained by a transformation $\vartheta$ of the above form can be described in the following way: Let $g: \mathbb{R} \longrightarrow \mathbb{R}$ be symmetric with respect to $\pi$, i.e., $g(\varphi)=g(2 \pi-\varphi), 2 \pi$-periodic, nonnegative, and $L^{1}$-integrable. Further, let $\tau(\varphi)$ be a real trigonometric polynomial of degree $N / 2$ with $N$ simple zeros in $(0,2 \pi)$, and let $L \in \mathbb{R}^{+}$be defined by

$$
L \leq \min \left\{|\tau(\varphi)|: \tau^{\prime}(\varphi)=0\right\} .
$$

Suppose that $R$ and $W$ are given as in Proposition 2. Then the weight function is given by

$$
\begin{cases}\frac{W\left(e^{i \varphi}\right)}{\sqrt{R\left(e^{i \varphi}\right)}} g(\hat{\tau}(\varphi)), & \varphi \in E_{N}, \\ 0, & \varphi \in[0,2 \pi] \backslash E_{N}\end{cases}
$$

where $\hat{\tau}(\varphi):=(\pi / L) \tau(\varphi)$, fits into the class of measures treated in this paper. In fact, define the new weight function as

$$
f_{0}(\varphi):=g\left(\pi \cos \frac{\varphi}{2}\right), \quad \varphi \in[0,2 \pi]
$$


Then for $\varphi \in E_{N}$,

$$
\begin{aligned}
f(\varphi) & :=\frac{W\left(e^{i \varphi}\right)}{\sqrt{R\left(e^{i \varphi}\right)}} f_{0}(\vartheta(\varphi))=\frac{W\left(e^{i \varphi}\right)}{\sqrt{R\left(e^{i \varphi}\right)}} g\left(\pi \cos \frac{\vartheta(\varphi)}{2}\right) \\
& =\frac{W\left(e^{i \varphi}\right)}{\sqrt{R\left(e^{i \varphi}\right)}} g(\hat{\tau}(\varphi))
\end{aligned}
$$

where the last identity follows by Proposition 1(h).

For some further illustrative applications for constructing new orthogonality measures by using the transformation $\Theta(z)$, see Section 4 .

\section{Main results}

In the previous section, we introduced a transformation of measures, respectively, C-functions. In this section, we will see that these transformations, given as in (2.6), lead exactly to all the reflection coefficients considered in (1.7).

The following theorem shows how the monic orthogonal polynomials $P_{n}\left(z, \sigma_{0}\right)$ and $P_{n N}(z, \sigma)$ are related to each other.

Theorem 2. Let $\left\{P_{n}\left(z, \sigma_{0}\right)\right\}$ be a sequence of orthogonal polynomials with respect to the symmetric measure $\sigma_{0}$, and let the function $\Theta$ be of the form (2.6). Under the assumptions of Proposition 2, the following relations hold for every $n \in \mathbb{N}_{0}$ :

$$
\begin{aligned}
P_{n N}(z, \sigma)=\kappa_{n}\left(\frac{z^{N}}{\Theta(z)}\right)^{n / 2}\left\{P_{n}\left(\Theta(z), \sigma_{0}\right)(\gamma\right. & \left.+\frac{\sqrt{R(z)}}{W(z)}\right) \\
& \left.+P_{n}^{*}\left(\Theta(z), \sigma_{0}\right)\left(\gamma-\frac{\sqrt{R(z)}}{W(z)}\right)\right\}
\end{aligned}
$$

and the polynomials of the second kind can be represented in the form

$$
\begin{aligned}
\Omega_{n N}(z, \sigma)=\kappa_{n}\left(\frac{z^{N}}{\Theta(z)}\right)^{n / 2} & \left\{\Omega_{n}\left(\Theta(z), \sigma_{0}\right)\left(1+\gamma \frac{W(z)}{\sqrt{R(z)}}\right)\right. \\
+ & \left.\Omega_{n}^{*}\left(\Theta(z), \sigma_{0}\right)\left(1-\gamma \frac{W(z)}{\sqrt{R(z)}}\right)\right\}+i \operatorname{Im} \gamma \cdot P_{2 n N}(z, \sigma) .
\end{aligned}
$$

Here, the constant $\kappa_{n}$ is given by $\kappa_{n}=\frac{L^{n}}{2^{n+1} \gamma \alpha^{n}}$.

The formulas in Theorem 2 can be used to derive asymptotics for the polynomials $\left\{P_{n}(z, \sigma)\right\}$ from the asymptotic behavior of the original polynomials $\left\{P_{n}\left(z, \sigma_{0}\right)\right\}$. To illustrate this in the following corollary, we consider a measure $\sigma_{0}$ in the Szegö-class, i.e., $d \sigma_{0}(\varphi)=p(\varphi) d \varphi+d \sigma_{0, s}(\varphi)$ where $\sigma_{0, s}$ is the singular part in the Lebesgue decomposition and where $p(\varphi)$ is a positive integrable function which satisfies Szegö's condition

$$
\int_{0}^{2 \pi} \ln p(\varphi) d \varphi>-\infty
$$

Then it is known, e.g. [23, Thm.12.1.1] and [8, Thm.21.1], that the orthonormal polynomials $\left\{\Phi_{n}\left(z, \sigma_{0}\right)=k_{n}\left(\sigma_{0}\right) z^{n}+\cdots\right\}$, normalized such that

$$
\int_{0}^{2 \pi}\left|\Phi_{n}\left(e^{i \varphi}, \sigma_{0}\right)\right|^{2} d \sigma_{0}(\varphi)=1, \quad k_{n}\left(\sigma_{0}\right)>0
$$


satisfy

$$
\begin{aligned}
& \lim _{n \rightarrow \infty} \Phi_{n}\left(z, \sigma_{0}\right)=0, \\
& \lim _{n \rightarrow \infty} \Phi_{n}^{*}\left(z, \sigma_{0}\right)=\frac{1}{D\left(z, \sigma_{0}\right)}
\end{aligned}
$$

uniformly on compact subsets on $\{|z|<1\}$ and

$$
\lim _{n \rightarrow \infty} k_{n}\left(\sigma_{0}\right)=\frac{1}{D\left(0, \sigma_{0}\right)}
$$

Here, $D\left(z, \sigma_{0}\right)$ is the so-called Szegö-function defined by

$$
D\left(z, \sigma_{0}\right)=\exp \left\{\frac{1}{4 \pi} \int_{0}^{2 \pi} \frac{e^{i \varphi}+z}{e^{i \varphi}-z} \ln p(\varphi) d \varphi\right\}, \quad|z|<1 .
$$

Corollary 1. Let the assumptions of Theorem 2 be fulfilled and suppose that $\Gamma_{E_{N}}$ is a strict subset of the unit circle. Further, let $\left\{\Phi_{n}(z, \sigma)\right\}$ and $\left\{\Phi_{n}\left(z, \sigma_{0}\right)\right\}$ be the orthonormal polynomials with respect to the measures $\sigma$ and $\sigma_{0}$, respectively. If the measure $\sigma_{0}$ belongs to the Szegö-class, then the asymptotic result

$$
\lim _{n \rightarrow \infty} 2 \gamma \sqrt{\operatorname{Re} \gamma} \alpha^{n}\left(\frac{\Theta(z)}{z^{N}}\right)^{n / 2} \Phi_{n N}(z, \sigma)=D\left(\Theta(z), \sigma_{0}\right)^{-1}\left(\gamma-\frac{\sqrt{R(z)}}{W(z)}\right)
$$

holds uniformly in compact subsets on $\mathbb{C} \backslash \Gamma_{E_{N}}$.

Remark. Similar investigations as for Szegö measures $\sigma_{0}$ also can be made for orthogonal polynomials with asymptotically periodic reflection coefficients, $\mathrm{cf}$. $[2,3,10,20]$.

If we combine the asymptotic relation in the above corollary with formula (4.2) below, taking into consideration that the polynomials $P_{\nu}^{(n N)}$ and $\hat{\Omega}_{\nu}^{(n N)}$ in (4.2) are dependent on $n$ in a known way (compare Theorem 3 below), then we immediately get asymptotics not only for the subsequence $\left\{\Phi_{n N}(z, \sigma)\right\}$ but for the whole sequence of orthogonal polynomials $\left\{\Phi_{n}(z, \sigma)\right\}$.

The next theorem gives the announced effects of the transformation $\Theta$ on the reflection coefficients of the orthogonal polynomials $P_{n}(z, \sigma)$.

Theorem 3. Let $P_{n}(z, \sigma)$ be the orthogonal polynomials with respect to the measure $\sigma$, defined as in Proposition 2, and let $\alpha,|\alpha|=1$ be the leading coefficient of the polynomial $\mathcal{T}$. Then there holds:

(a) The reflection coefficients $a_{0}(\sigma), \ldots, a_{N-2}(\sigma)$ only depend on the polynomials $R, \mathcal{T}$, and $W$ (and not on the measure $\sigma_{0} ;$ for calculation, compare the lines after Theorem 5).

(b) For all $n \in \mathbb{N}_{0}$, we have

$$
a_{n N+j}(\sigma)=\alpha^{2 n} a_{j}(\sigma), \quad j=0,1, \ldots, N-2,
$$

and

$$
a_{(n+1) N-1}(\sigma)=\frac{\alpha^{2(n+1)}}{2}\left[a_{n}\left(\sigma_{0}\right)\left(\gamma^{2}+1\right)+\gamma^{2}-1\right]
$$

The statements of Theorem 3 simplify if we additionally suppose that $\mathcal{T}$ is a monic polynomial, i.e., that $\alpha=1$. Let us point out that $\alpha=1$ does not imply that $\mathcal{T}$ is 
a real polynomial in general. Then, Theorem 3 says that the sequence of reflection coefficients $\left\{a_{n}(\sigma)\right\}$ is "nearly" periodic, i.e., we have

$$
\begin{aligned}
\ldots, \underbrace{a_{0}(\sigma), \ldots, a_{N-2}(\sigma)}, a_{n N-1}(\sigma), \underbrace{a_{0}(\sigma), \ldots, a_{N-2}(\sigma)}, a_{(n+1) N-1}(\sigma), & \underbrace{a_{0}(\sigma), \ldots, a_{N-2}(\sigma)}, \ldots
\end{aligned}
$$

where only the $a_{n-1}(\sigma)$ 's depend on $n$. In the simplest case, when $\alpha=1$ and $\gamma=W(0) / \sqrt{R(0)}=1$, we further have

$$
a_{(n+1) N-1}(\sigma)=a_{n}\left(\sigma_{0}\right)
$$

i.e., the new orthogonal polynomials arise from the old ones by plugging in the fixed sequence $a_{0}(\sigma), \ldots, a_{N-2}(\sigma)$ between each pair of successive reflection coefficients $a_{n}\left(\sigma_{0}\right)$ and $a_{n+1}\left(\sigma_{0}\right)$.

If $\alpha=\gamma=1$, one can say even more about the values of the reflection coefficients $a_{0}(\sigma), \ldots, a_{N-2}(\sigma)$.

Corollary 2. Suppose that $\alpha=1$, and $\gamma=W(0) / \sqrt{R(0)}=1$. Then, under the assumptions of Theorem 3, there holds:

$$
a_{N-2-j}(\sigma)=-\overline{a_{j}(\sigma)} \quad \text { for } j=0,1, \ldots, N-2 .
$$

Finally, let us show that also the converse of Corollary 2 holds. This will complete the correspondence in (1.7).

Theorem 4. Let $\left\{a_{n}(\sigma)\right\}$ be a sequence of complex numbers with the following properties:

(1) $\left|a_{n}(\sigma)\right|<1$ for all $n \in \mathbb{N}$,

(2) $\quad a_{N-2-j}(\sigma)=-\overline{a_{j}(\sigma)}, \quad j=0,1, \ldots, N-2$,

(3) $\quad a_{n}(\sigma)=a_{n+N}(\sigma)$ for all $n \in \mathbb{N}_{0} \backslash\{k N-1: k \in \mathbb{N}\}$,

(4) $a_{n N-1}(\sigma) \in(-1,1)$ for all $n \in \mathbb{N}$,

$N \in \mathbb{N}$ fixed. Further, let $\sigma_{0}$ be the measure associated with the reflection coefficients $\left\{a_{n}\left(\sigma_{0}\right)\right\}$ where $a_{n}\left(\sigma_{0}\right):=a_{(n+1) N-1}(\sigma)$ for all $n \in \mathbb{N}_{0}$. Then there holds:

(i) The orthogonal polynomials $\left\{P_{n}(z, \sigma)\right\}$, generated by the reflection coefficients $\left\{a_{n}(\sigma)\right\}$, and the orthogonal polynomials $\left\{P_{n}\left(z, \sigma_{0}\right)\right\}$, generated by the reflection coefficients $\left\{a_{n}\left(\sigma_{0}\right)\right\}$, are related as in Theorem 2. Furthermore, the measure $\sigma$ is of the form (2.11).

(ii) The transformation $\Theta$, respectively the polynomials $R, W$, and $\mathcal{T}$, are given by

$$
\begin{aligned}
R(z) & =\left(P_{N-1}^{*}(z, \sigma)-z P_{N-1}(z, \sigma)\right) \cdot W(z), \\
W(z) & =\hat{\Omega}_{N-1}^{*}(z, \sigma)-z \hat{\Omega}_{N-1}(z, \sigma), \\
\mathcal{T}(z) & =z P_{N-1}(z, \sigma)+P_{N-1}^{*}(z, \sigma),
\end{aligned}
$$

where $\hat{\Omega}_{N-1}(z, \sigma)$ denotes the monic polynomial of the second kind with respect to $\sigma$.

\section{Examples and some further associated results}

Let us give some illustrative applications for constructing new orthogonal polynomials, respectively, measures by using the transformation $\Theta(z)$ : 
Example. (a) The special setting

$$
\mathcal{T}(z)=z^{N}+1, \quad R(z)=\left(z^{N}-1\right)^{2}, \quad W(z)=\sqrt{R(z)}=1-z^{N}
$$

gives $\Theta(z)=z^{N}$, and the new polynomials $P_{n N}(z, \sigma)$ are of the simple form

$$
P_{n N}(z, \sigma)=P_{n}\left(z^{N}, \sigma_{0}\right)
$$

Further,

$$
\sigma(\varphi)=\frac{1}{N} \sigma_{0}(N \varphi)
$$

and, by applying Theorem 1, it is easy to see that the remaining polynomials are given by

$$
P_{n N+j}(z, \sigma)=z^{j} P_{n}\left(z^{N}, \sigma_{0}\right), \quad j=1, \ldots, N-1 .
$$

Hence, the new sequence of reflection coefficients $\left\{a_{n}(\sigma)\right\}$ results from the original one by inserting $N-1$ zeros between each pair of successive reflection coefficients $a_{k}\left(\sigma_{0}\right)$ and $a_{k+1}\left(\sigma_{0}\right)$, i.e.,

$$
\left\{a_{n}(\sigma)\right\}=\{\ldots, \underbrace{0, \ldots, 0}_{N-1 \text { times }}, a_{k}\left(\sigma_{0}\right), \underbrace{0, \ldots, 0}_{N-1 \text { times }}, a_{k+1}\left(\sigma_{0}\right), \underbrace{0, \ldots, 0}_{N-1 \text { times }}, \ldots\} .
$$

(b) Let $\mu_{0}$ be a positive Borel measure on the real interval $[-1,1]$ and let $\left\{p_{n}\left(x, \mu_{0}\right)\right\}$ be the corresponding orthogonal polynomials. Suppose that $T$ is a real polynomial of degree $N$ such that

$$
E:=\{x \in \mathbb{R}:|T(x)| \leq 1\}=\bigcup_{j=1}^{N}\left[\alpha_{2 j-1}, \alpha_{2 j}\right],
$$

$\alpha_{1}=-1$ and $\alpha_{2 N}=1$, is a set of $N$ intervals. Then Geronimo and Van Assche [9] have shown that the polynomials $p_{n}(T(x))$ are orthogonal with respect to the measure

$$
d \mu(x)= \begin{cases}\frac{V(x)}{T^{\prime}(x)} d \mu_{0}(T(x)), & x \in E, \\ 0, & x \in[-1,1] \backslash E\end{cases}
$$

where $V$ is a real polynomial of degree $N-1$ which has exactly one zero in each gap $\left[\alpha_{2 j}, \alpha_{2 j+1}\right], j=1, \ldots, N-1$. Let us consider the additional conditions that $d \mu_{0}(x)=\omega_{0}(x) d x$ where $\omega_{0}$ is of the form $\omega_{0}(x)=\tilde{\omega}_{0}\left(a x^{2}+b\right), a, b \in \mathbb{R}, a \neq 0$ and that $V$ has only zeros which lie at the boundary points of the gaps. Then it follows with the help of the well-known relationship $\sigma(\varphi)=\mu(\cos \varphi)$ between orthogonality measures of polynomials orthogonal on $[-1,1]$ and on $[0,2 \pi]$, respectively (see e.g. $[6,8,23])$, that $\mu_{0}^{\prime}(\cos \varphi)|\sin \varphi|$ is of the form $(2.12)$ where $\tau(\varphi)=T(\cos \varphi)$ and $W\left(e^{i \varphi}\right)=e^{i N \varphi} \sin \varphi V(\cos \varphi)$. Thus the polynomials orthogonal with respect to $\mu_{0}$ can be represented with the help of the polynomials orthogonal on the unit circle treated in this paper.

(c) Let us now consider the case that the original polynomials $P_{n}\left(z, \sigma_{0}\right)$ have periodic reflection coefficients, say with period $M$, i.e., $a_{n+M}\left(\sigma_{0}\right)=a_{n}\left(\sigma_{0}\right)$. Then it is known, cf. [7] or [19], that $\sigma_{0}$ essentially lives on $M$ arcs

$$
\Gamma_{E_{M}^{0}}:=\left\{e^{i \varphi}: \varphi \in E_{M}^{0}\right\} \quad \text { where } \quad E_{M}^{0}:=\bigcup_{j=1}^{M}\left[\varphi_{2 j-1}^{0}, \varphi_{2 j}^{0}\right]
$$


and $\varphi_{1}^{0} \leq \varphi_{2}^{0} \leq \cdots \leq \varphi_{2 M}^{0}$.

If we transform the periodic measure $\sigma_{0}$ in the way as given in Proposition 2, then the new sequence of orthogonal polynomials $\left\{P_{n}(z, \sigma)\right\}$ has again periodic reflection coefficients, now with period $N M$, and

$$
\left\{e^{i \varphi}: \varphi \in(\operatorname{supp}(\sigma))^{\prime}\right\}=\Theta^{-1}\left(\Gamma_{E_{M}^{0}}\right)=: \Gamma_{E_{N M}}
$$

consists of $N M$ arcs.

By Theorem 2, we know an exact representation of the subsequence $\left\{P_{n N}(z, \sigma)\right\}$ of the new orthogonal polynomials in terms of the original orthogonal polynomials $P_{n}\left(z, \sigma_{0}\right)$.

For the description of the orthogonal polynomials $P_{k}(z, \sigma)$, where $k$ is not necessarily a multiple of $N$, we will need the so-called associated polynomials.

Definition. Given a sequence $\left\{P_{n}(z, \mu)\right\}$ of orthogonal polynomials on the unit circle, then the $m$-th, $m \in \mathbb{N}_{0}$, monic associated polynomials, denoted by $P_{k}^{(m)}(z, \mu)$ and $\hat{\Omega}_{k}^{(m)}(z, \mu)$, respectively, introduced and studied by the first author [16], are given by $P_{0}^{(m)}(z, \mu)=\hat{\Omega}_{0}^{(m)}(z, \mu)=1$ and

$$
\begin{aligned}
& P_{k+1}^{(m)}(z, \mu)=z P_{k}^{(m)}(z, \mu)-\overline{a_{k+m}(\mu)} P_{k}^{(m) *}(z, \mu), \\
& \hat{\Omega}_{k+1}^{(m)}(z, \mu)=z \hat{\Omega}_{k}^{(m)}(z, \mu)+\overline{a_{k+m}(\mu)} \hat{\Omega}_{k}^{(m) *}(z, \mu)
\end{aligned}
$$

$k \in \mathbb{N}_{0}$. Note that $P_{k}^{(0)}(z, \mu)=P_{k}(z, \mu)$ and $\hat{\Omega}_{k}^{(0)}=\Omega_{k}(z, \mu) / F(0, \mu)$.

To obtain expressions for the polynomials $P_{n N+\nu}(z, \sigma), \nu \in\{0, \ldots, N-1\}$, we consider the identity (cf. [16, Cor.3.1])

$$
\begin{aligned}
2 P_{n N+\nu}(z, \sigma)=\left(P_{n N}(z, \sigma)+P_{n N}^{*}(z, \sigma)\right) P_{\nu}^{(n N)}(z, \sigma) & \\
& +\left(P_{n N}(z, \sigma)-P_{n N}^{*}(z, \sigma)\right) \hat{\Omega}_{\nu}^{(n N)}(z, \sigma) .
\end{aligned}
$$

By Theorem 2, the expressions $P_{n N}(z, \sigma) \pm P_{n N}^{*}(z, \sigma)$ can be written in terms of the original orthogonal polynomials $P_{n}:=P_{n}\left(\cdot, \sigma_{0}\right)$, i.e.,

$$
\begin{aligned}
P_{n N}(z, \sigma) \pm P_{n N}^{*}(z, \sigma)=\frac{1}{2}\left(\frac{L z^{N / 2}}{2 \sqrt{\Theta(z)}}\right)^{n} & {\left[\left(\bar{\alpha}^{n} \pm \alpha^{n}\right)\left(P_{n}+P_{n}^{*}\right)(\Theta(z))\right.} \\
+ & \left.\frac{\sqrt{R(z)}}{W(z)}\left(\bar{\alpha}^{n} \bar{\gamma} \mp \alpha^{n} \gamma\right)\left(P_{n}-P_{n}^{*}\right)(\Theta(z))\right]
\end{aligned}
$$

Hence, in order to make use of (4.2) for the explicit calculation of the orthogonal polynomials $P_{n N+\nu}(z, \sigma)$, we need information on $P_{\nu}^{(n N)}(z, \sigma)$ and $\hat{\Omega}_{\nu}^{(n N)}(z, \sigma)$, which are determined by the reflection coefficients $a_{n N}(\sigma), \ldots, a_{n N+\nu-1}(\sigma)$. What we are going to do is the following: Theorem 5 below gives an explicit representation of $P_{N}^{(n N)}(z, \sigma)$. If one knows $P_{N}^{(n N)}(z, \sigma)$, one easily can calculate the reflection coefficients $a_{n N}(\sigma), \ldots, a_{(n+1) N-1}(\sigma)$ by using a method introduced by Geronimus [8, Thm.9.2]: One has

$$
a_{(n+1) N-1}(\sigma)=-\overline{P_{N}^{(n N)}(0, \sigma)}
$$


and by (4.1)

$$
P_{N-1}^{(n N)}(z, \sigma)=\frac{P_{N}^{(n N)}(z, \sigma)+\overline{a_{(n+1) N-1}(\sigma)} P_{N}^{(n N) *}(z, \sigma)}{z\left(1-\left|a_{(n+1) N-1}(\sigma)\right|^{2}\right)} .
$$

Iterating this procedure gives the desired polynomials for their respective reflection coefficients.

Before we give an explicit representation of $P_{N}^{(n N)}(z, \sigma)$, we prove the following relationship between the reflection coefficients $\left\{a_{n}(\sigma)\right\}$ and $\left\{a_{n}\left(\sigma_{0}\right)\right\}$, which is also used in the proof of Theorem 5.

Corollary 3. The following relation holds:

$$
\prod_{j=n N}^{(n+1) N-1}\left(1-\left|a_{j}(\sigma)\right|^{2}\right)=\frac{L^{2}}{4}\left(1-a_{n}^{2}\left(\sigma_{0}\right)\right) \quad \text { for all } n \in \mathbb{N}_{0} .
$$

Now we can state

Theorem 5. For every $n \in \mathbb{N}_{0}$, there holds

$$
P_{N}^{(n N)}(z, \sigma)=\beta_{n} \mathcal{T}(z)+\delta_{n} W(z)+\lambda_{n} \frac{R(z)}{W(z)}
$$

where the constants $\gamma_{n}, \delta_{n}$, and $\lambda_{n}$ only depend on the reflection coefficient $a_{n}\left(\sigma_{0}\right)$ and are given by

$$
\begin{aligned}
\beta_{n} & =\frac{i \operatorname{Im}\left\{\alpha^{n}\right\}\left(a_{n}\left(\sigma_{0}\right)+1\right)-\gamma \operatorname{Re}\left\{\alpha^{n} \gamma\right\}\left(a_{n}\left(\sigma_{0}\right)-1\right)}{2 \alpha^{n+1} \gamma \operatorname{Re} \gamma} \\
\delta_{n} & =\frac{i \operatorname{Im}\left\{\alpha^{n}\right\}\left(a_{n}\left(\sigma_{0}\right)-1\right)}{2 \alpha^{n+1} \operatorname{Re} \gamma} \\
\lambda_{n} & =\frac{-\operatorname{Re}\left\{\alpha^{n} \gamma\right\}\left(a_{n}\left(\sigma_{0}\right)+1\right)}{2 \alpha^{n+1} \gamma \operatorname{Re} \gamma}
\end{aligned}
$$

Here, $\alpha$ and $\gamma$ are defined as in (2.7) and (2.10), respectively.

For the calculation of the reflection coefficients $a_{0}(\sigma), \ldots, a_{N-2}(\sigma)$, we consider two cases:

- Special case: For $\Theta(z)=z^{N}$, we have $a_{0}(\sigma)=a_{1}(\sigma)=\cdots=a_{N-2}(\sigma)=0$, and hence, $P_{n N+j}(z, \sigma)=z^{j} P_{n}\left(z^{N}, \sigma_{0}\right), n \in \mathbb{N}_{0}, j=0, \ldots, N-1$; compare Example (a) at the beginning of this section.

- General case: By Theorem 3, the reflection coefficients $a_{0}(\sigma), \ldots, a_{N-2}(\sigma)$ only depend on $\mathcal{T}, R$, and $W$, i.e., only on the transformation $\Theta$. In particular, they do not depend on the measure $\sigma_{0}$. Or, in other words, for arbitrary $\sigma_{0}$ and $\tilde{\sigma}_{0}$, we have

$$
\begin{aligned}
z P_{N-1}^{(n N)}(z, \tilde{\sigma}) & =z P_{N-1}^{(n N)}(z, \sigma) \\
& =\frac{P_{N}^{(n N)}(z, \sigma)-P_{N}^{(n N)}(0, \sigma) P_{N}^{(n N) *}(z, \sigma)}{1-\left|P_{N}^{(n N)}(0, \sigma)\right|^{2}}
\end{aligned}
$$

where $\tilde{\sigma}$ is the distribution function associated with $\tilde{\sigma}_{0}$ by (2.10). Hence, let us take the simplest distribution $\sigma_{0}(\varphi)=\varphi$, i.e., $a_{n}\left(\sigma_{0}\right)=0$ for all $n \in \mathbb{N}_{0}$. Then Theorem 5 yields

$$
P_{N}(z, \sigma)=\beta_{0} \mathcal{T}(z)+\lambda_{0} \frac{R(z)}{W(z)}
$$


with

$$
\beta_{0}=\frac{1}{2 \alpha} \quad \text { and } \quad \lambda_{0}=-\frac{1}{2 \alpha \gamma} .
$$

Using relation (4.4) with $n=0$ successively, we obtain the desired reflection coefficients $a_{0}(\sigma), \ldots, a_{N-2}(\sigma)$.

Remark. (Compare Example (b) at the beginning of this section). Let $\mu_{0}$ be a measure on the real interval $[-1,1]$, and let the polynomials $T, V$ and the set $E$ be such as in Example (b). There we have shown that (under certain additional assumptions) the polynomials $p_{n}(x, \mu)$ orthogonal with respect to the measure $d \mu(x)=$ $\left(V(x) / T^{\prime}(x)\right) d \mu_{0}(T(x))$ can be represented with the help of the polynomials studied in this paper. Let $\left\{\alpha_{n}(\mu)\right\}$ and $\left\{\lambda_{n}(\mu)\right\}$ denote the recurrence coefficients of the orthogonal polynomials $\left\{p_{n}(x, \mu)\right\}$, i.e.,

$$
p_{n}(x, \mu)=\left(x-\alpha_{n}(\mu)\right) p_{n-1}(x, \mu)-\lambda_{n}(\mu) p_{n-2}(x, \mu) .
$$

It has been shown by Geronimo and Van Assche [9, Thm.6, formula (3.7)] that the $\alpha_{n N+1}(\mu)$ 's, $n \in \mathbb{N}_{0}$ are independent of $\mu_{0}$ and that $\alpha_{n N+1}(\mu)=\alpha_{(n+1) N+1}(\mu)$. But, in fact, under the additional conditions posed in Example (b), much more holds. Indeed, by the well-known connection of the recurrence coefficients of real orthogonal polynomials and those of the corresponding orthogonal polynomials on the unit circle, cf. [8, Thm.31.1,p.67], we even obtain, by applying Theorem 3 , that

- for $N \geq 2: \alpha_{n N+2}(\mu), \ldots, \alpha_{(n+1) N}(\mu), n \in \mathbb{N}$ are independent of $\mu_{0}$ and satisfy

$$
\alpha_{n N+j}(\mu)=\alpha_{(n+1) N+j}(\mu), \quad j=2, \ldots, N,
$$

- for $N \geq 3: \lambda_{n N+3}(\mu), \ldots, \lambda_{(n+1) N}(\mu), n \in \mathbb{N}$ are independent of $\mu_{0}$ and satisfy

$$
\lambda_{n N+j}(\mu)=\lambda_{(n+1) N+j}(\mu), \quad j=3, \ldots, N
$$

\section{Proofs}

Proof of Proposition 1. The analyticity of $\Theta(z)$ follows immediately from definition (2.6). Furthermore, by (2.3), we can write

$$
\frac{\Theta(z)}{z^{N}}=\frac{L^{2}}{(\mathcal{T}(z)+\sqrt{R(z)})^{2}}
$$

and (2.7) gives the assertion in (a). Part (b), (c), and (d) can be derived from [20, Lemma 2.1], where one has to take into consideration that the notation in [20] is slightly different from the one used here; see Remark before (2.6). Parts (e) and (g) follow from the definition of the polynomials $\mathcal{T}$ and $R$ and from (2.8). Choosing a fixed branch of the $N$-root, the function $Y(z)$ is analytic on $\mathbb{C} \backslash \Gamma_{E_{N}}$. By the already proven parts, we know that $Y$ has no zeros on $\mathbb{C} \backslash \Gamma_{E_{N}}$, that it maps $\mathbb{C} \backslash \Gamma_{E_{N}}$ onto $\{|z|>1\}$, and that $|Y(z)|=1$ on $\Gamma_{E_{N}}$. Now recall that $|Y(z) / z|=\mathcal{O}(1)$ for $z \rightarrow \infty$. Altogether this proves part (f). In order to get part (h), let us recall that on the one hand we have

and on the other hand

$$
\sqrt{\Theta\left(e^{i \varphi}\right)}=e^{i \vartheta(\varphi) / 2}=\cos \frac{\vartheta(\varphi)}{2}+i \sin \frac{\vartheta(\varphi)}{2}
$$

$$
\sqrt{\Theta\left(e^{i \varphi}\right)}=\frac{\mathcal{T}\left(e^{i \varphi}\right)-\sqrt{R\left(e^{i \varphi}\right)}}{L e^{i(N / 2) \varphi}}
$$


Hence, the assertion follows from (2.8) and (2.9).

Proof of Proposition 2. Let us abbreviate

$$
\mathcal{G}(z):=\frac{W(z)}{\sqrt{R(z)}} F\left(\Theta(z), \sigma_{0}\right) .
$$

By the assumptions on $W, R$, and $\Theta$, the function $\mathcal{G}(z)$ is analytic on $\mathbb{C} \backslash \Gamma_{E_{N}}$. In order to see that $\mathcal{G}(z)-i \operatorname{Im} \gamma$ is a C-function, it remains to show that

$$
\mathcal{G}(0)-i \operatorname{Im} \gamma \in \mathbb{R}^{+}
$$

and

$$
\operatorname{Re}\{\mathcal{G}(z)-i \operatorname{Im} \gamma\}>0 \quad \text { on }|z|<1 .
$$

Indeed, we will prove the representation

$$
\mathcal{G}(z)-i \operatorname{Im} \gamma=\int_{0}^{2 \pi} \frac{e^{i \varphi}+z}{e^{i \varphi}-z} d \sigma(\varphi)
$$

where $\sigma$ is given as in the statement of our Proposition. Then

$$
\operatorname{Re} \gamma=\mathcal{G}(0)-i \operatorname{Im} \gamma=\int_{0}^{2 \pi} d \sigma(\varphi)>0
$$

(this will be needed later in the proof of Corollary 3) and for $z=s e^{i \alpha}, s \in[0,1)$,

$$
\operatorname{Re}\{\mathcal{G}(z)-i \operatorname{Im} \gamma\}=\int_{0}^{2 \pi} \frac{1-s^{2}}{1-2 s \cos (\varphi-\alpha)+s^{2}} d \sigma(\varphi)>0
$$

since $\sigma$ is nonnegative and does not vanish identically.

In order to prove (5.1), let us consider the functions

$$
\mathcal{F}_{s}(z):=\frac{W(z)}{\sqrt{R(z)}} F\left(s \Theta(z), \sigma_{0}\right)-i \operatorname{Im} \gamma, \quad s \in[0,1), \quad z \in \mathbb{C} \backslash \Gamma_{E_{N}} .
$$

For every fixed $s \in[0,1)$, the boundary values $\mathcal{F}_{s}\left(e^{i \varphi}\right):=\lim _{t \rightarrow 1^{-}} \mathcal{F}_{s}\left(t e^{i \varphi}\right)$ exist for all $\varphi \in[0,2 \pi]$, except of the poles of $W\left(e^{i \varphi}\right) / \sqrt{R\left(e^{i \varphi}\right)}$ and $\mathcal{F}_{s} \in H_{p}=H_{p}(\{|z|<1\})$, $p \in[1,2)$ where $H_{p}$ denotes the Hardy space (see, e.g., [5, Theorem 3.2]). Hence, by a strong version of Schwarz' formula, we can write, cf. [13, Chapter I.D and V.B] or [16, Lemma 2.1],

$$
\begin{aligned}
\mathcal{F}_{s}(z) & =\frac{1}{2 \pi} \int_{0}^{2 \pi} \frac{e^{i \varphi}+z}{e^{i \varphi}-z} \operatorname{Re} \mathcal{F}_{s}\left(e^{i \varphi}\right) d \varphi \\
& :=\int_{0}^{2 \pi} \frac{e^{i \varphi}+z}{e^{i \varphi}-z} d \sigma_{s}(\varphi), \quad|z|<1,
\end{aligned}
$$

i.e., the family $\left\{\sigma_{s}\right\}_{s \in[0,1)}$ is defined by

$$
\sigma_{s}(\psi):=\frac{1}{2 \pi} \int_{0}^{\psi} \operatorname{Re} \mathcal{F}_{s}\left(e^{i \varphi}\right) d \varphi, \quad \psi \in[0,2 \pi] .
$$

For all $\varphi \in\left(\varphi_{2 j-1}, \varphi_{2 j}\right), j=1, \ldots, N$, there holds

$$
\operatorname{Re} \mathcal{F}_{s}\left(e^{i \varphi}\right)=\frac{W\left(e^{i \varphi}\right)}{\sqrt{R\left(e^{i \varphi}\right)}} \operatorname{Re} F\left(s \Theta\left(e^{i \varphi}\right), \sigma_{0}\right)>0
$$


since $F\left(z, \sigma_{0}\right)$ is a C-function and $W\left(e^{i \varphi}\right) / \sqrt{R\left(e^{i \varphi}\right)}>0$ on $\left(\varphi_{2 j-1}, \varphi_{2 j}\right)$. If $\varphi \notin E_{N}$, we have

$$
\operatorname{Re} \mathcal{F}_{s}\left(e^{i \varphi}\right)=\frac{i W\left(e^{i \varphi}\right)}{\sqrt{R\left(e^{i \varphi}\right)}} \operatorname{Im} F\left(s \Theta\left(e^{i \varphi}\right), \sigma_{0}\right)=0
$$

because $s \Theta\left(e^{i \varphi}\right) \in[0, s]$ by Proposition $1(\mathrm{~d})$ and $F\left(x, \sigma_{0}\right) \in \mathbb{R}$ for all $x \in[0,1)$. Hence, for $\psi \in\left[\varphi_{2 j-1}, \varphi_{2 j}\right]$, we can write

$$
\begin{aligned}
\sigma_{s}(\psi) & =\sigma_{s}\left(\varphi_{2 j-1}\right)+\frac{1}{2 \pi} \int_{\varphi_{2 j-1}}^{\psi} \frac{W\left(e^{i \varphi}\right)}{\sqrt{R\left(e^{i \varphi}\right)}} \operatorname{Re} F\left(s \Theta\left(e^{i \varphi}\right), \sigma_{0}\right) d \varphi \\
& =\sigma_{s}\left(\varphi_{2 j-1}\right)+\frac{1}{2 \pi} \int_{0}^{\vartheta(\psi)} \frac{W\left(e^{i \vartheta_{j}^{-1}(\varphi)}\right)}{\sqrt{R\left(e^{i \vartheta_{j}^{-1}(\varphi)}\right)}} \frac{\operatorname{Re} F\left(s e^{i \varphi}, \sigma_{0}\right)}{\vartheta^{\prime}\left(\vartheta_{j}^{-1}(\varphi)\right)} d \varphi
\end{aligned}
$$

where $\vartheta_{j}:=\left.\vartheta\right|_{\left[\varphi_{2 j-1}, \varphi_{2 j}\right]}$.

Next we claim that for all $\eta \in E_{N}$, the expression

$$
\frac{W\left(e^{i \eta}\right)}{\sqrt{R\left(e^{i \eta}\right)}} \cdot \frac{1}{\vartheta^{\prime}(\eta)}=\left|\frac{w(\eta)}{\sqrt{-\rho(\eta)}}\right| \cdot \frac{1}{\vartheta^{\prime}(\eta)}
$$

where $w(\eta):=i e^{-i(N / 2) \eta} W\left(e^{i \eta}\right)$, is bounded on $E_{N}$. For that, we write explicitly

$$
\begin{aligned}
\vartheta^{\prime}(\eta) & =\left(\arctan \frac{\sqrt{-\rho(\eta)}}{\tau(\eta)}\right)^{\prime} \\
& =\frac{\frac{-\rho^{\prime}(\eta)}{2 \sqrt{-\rho(\eta)}} \tau(\eta)-\sqrt{-\rho(\eta)} \tau^{\prime}(\eta)}{\tau^{2}(\eta)-\rho(\eta)}=-\frac{\rho^{\prime}(\eta) \tau(\eta)-2 \rho(\eta) \tau^{\prime}(\eta)}{2 L^{2} \sqrt{-\rho(\eta)}} .
\end{aligned}
$$

This means that the term $\sqrt{-\rho(\eta)}$ cancels out in (5.5). Now we distinguish two cases for $\varphi \in E_{N}$, namely, if $\eta$ is a double zero of $\rho(\varphi)$ or not:

(i) In the first case, i.e., $\eta \neq \varphi_{2 j}=\varphi_{2 j+1}$, there holds:

$$
\rho^{\prime}(\eta) \tau(\eta) \neq 2 \rho(\eta) \tau^{\prime}(\eta)
$$

Recall that either $\tau^{\prime}(\eta)>0$ or $\tau^{\prime}(\eta)<0$ for all $\eta \in\left(\varphi_{2 j-1}, \varphi_{2 j}\right)$. Hence, if $\eta \in$ $\left(\varphi_{2 j-1}, \varphi_{2 j}\right)$ and $\tau^{\prime}(\eta)>0$, then $2 \rho(\eta) \tau^{\prime}(\eta)<0$ and $\rho^{\prime}(\eta) \tau(\eta) \geq 0$, respectively, $2 \rho(\eta) \tau^{\prime}(\eta)>0$ and $\rho^{\prime}(\eta) \tau(\eta) \leq 0$ if $\tau^{\prime}(\eta)<0$ on $\left(\varphi_{2 j-1}, \varphi_{2 j}\right)$. If $\eta=\varphi_{2 j-1}$ or $\eta=\varphi_{2 j} \neq \varphi_{2 j+1}$, then the left-hand side in (5.6) is unequal to zero and the righthand side is zero.

(ii) In the second case, i.e., $\eta=\varphi_{2 j}=\varphi_{2 j+1}$, it is easy to see that $\eta$ is a simple zero of $\rho^{\prime} \tau-2 \rho \tau^{\prime}$. However, then $\eta$ is also a zero of $w(\varphi)$.

Combining both cases gives the desired boundedness of the expression in (5.5).

According to (1.2), we now can apply Helly's theorem which gives the existence of a subsequence $\sigma_{s_{k}}, k \in \mathbb{N}$, with $s_{k} \rightarrow 1$ as $k \rightarrow \infty$ such that

$$
\sigma_{s_{k}} \rightarrow \sigma_{\infty}=: \sigma \quad \text { pointwise on }[0,2 \pi]
$$

and

$$
\lim _{k \rightarrow \infty} \int_{0}^{2 \pi} h(\varphi) d \sigma_{s_{k}}(\varphi)=\int_{0}^{2 \pi} h(\varphi) d \sigma(\varphi)
$$

for any continuous function $h$ on $[0,2 \pi]$.

Together with (5.3), (5.4), and (1.2), this proves the proposition. 
For the proof of Theorem 2, we will need the following.

Lemma 1. Let $Q$ be a real self-reversed polynomial, i.e., $Q= \pm Q^{*}$, of degree $n$, $n \in \mathbb{N}_{0}$. Then,

- if $Q=Q^{*}$,

$$
\mathcal{Q}(z):=\left(\frac{z^{N}}{\Theta(z)}\right)^{n / 2} Q(\Theta(z))
$$

is a polynomial of degree $n N$ and $\mathcal{Q}=\mathcal{Q}^{*}$

- if $Q=-Q^{*}$,

$$
\mathcal{Q}(z):=\left(\frac{z^{N}}{\Theta(z)}\right)^{n / 2} \sqrt{R(z)} Q(\Theta(z))
$$

is a polynomial of degree $(n+1) N, \mathcal{Q}=\mathcal{Q}^{*}$ and $\mathcal{Q} / R$ is a polynomial of degree $(n-1) N$.

Proof. Let us first consider the case $Q=Q^{*}$. We can write $\mathcal{Q}$ in the form

$$
\mathcal{Q}(z)=\left(\frac{z^{N / 2}}{\sqrt{\Theta(z)}}\right)^{n} \tilde{Q}(\sqrt{\Theta(z)}) \text { and } \tilde{Q}(z):=Q\left(z^{2}\right)
$$

where we take the "positive" branch of $\sqrt{\Theta(z)}$, i.e.,

$$
\sqrt{\Theta(z)}=\frac{\mathcal{T}(z)-\sqrt{R(z)}}{L z^{N / 2}}
$$

Then $\tilde{Q}$ is again a self-reversed polynomial and contains only even powers of $z$, i.e., $\tilde{Q}(z)=\sum_{j=0}^{n-1} b_{2 n-j}\left(z^{2 n-j}+z^{j}\right)+b_{n} z^{n}$ where all the $b_{\nu}$ 's with odd $\nu$ vanish. According to (2.3), we have

$$
\frac{z^{N / 2}}{\sqrt{\Theta(z)}}=\frac{\mathcal{T}(z)+\sqrt{R(z)}}{L}
$$

and thus

$$
\begin{aligned}
\mathcal{Q}(z)= & b_{n} z^{n N / 2}+\sum_{j=0}^{n-1} b_{2 n-j}\left(\frac{\mathcal{T}(z)+\sqrt{R(z)}}{L}\right)^{n} \\
& \times\left[\left(\frac{\mathcal{T}(z)-\sqrt{R(z)}}{L z^{N / 2}}\right)^{2 n-j}+\left(\frac{\mathcal{T}(z)-\sqrt{R(z)}}{L z^{N / 2}}\right)^{j}\right] \\
= & b_{n} z^{n N / 2}+\sum_{j=0}^{n-1} \frac{b_{2 n-j} z^{j N / 2}}{L^{n-j}}\left[(\mathcal{T}(z)-\sqrt{R(z)})^{n-j}+(\mathcal{T}(z)+\sqrt{R(z)})^{n-j}\right] \\
= & b_{n} z^{n N / 2}+2 \sum_{j=0}^{n-1} \frac{b_{2 n-j} z^{j N / 2}}{L^{n-j}} \sum_{\nu=0}^{\lfloor(n-j) / 2\rfloor}\left(\begin{array}{c}
n-j \\
2 \nu
\end{array}\right) \mathcal{T}^{n-j-2 \nu}(z) R^{\nu}(z) .
\end{aligned}
$$

Recall that $b_{\nu}=0$ if $\nu$ is odd and note that, for even $j$, the expression

$$
z^{j N / 2} \sum_{\nu=0}^{\lfloor(n-j) / 2\rfloor}\left(\begin{array}{c}
n-j \\
2 \nu
\end{array}\right) \mathcal{T}^{n-j-2 \nu}(z) R^{\nu}(z)
$$

is a polynomial of exact degree $n N-j N / 2$. Thus, $\mathcal{Q}$ is a polynomial of exact degree $n N$. In addition, from the last representation, we get that $\mathcal{Q}=\mathcal{Q}^{*}$. 
If $Q=-Q^{*}$, then $\tilde{Q}(z):=Q\left(z^{2}\right)=\sum_{j=0}^{n-1} b_{2 n-j}\left(z^{2 n-j}-z^{j}\right)$ where again $b_{\nu}=0$ if $\nu$ is odd. Similarly, as above, we can write

$$
\mathcal{Q}(z)=-2 R(z) \sum_{j=0}^{n-1} \frac{b_{2 n-j} z^{j N / 2}}{L^{n-j}} \sum_{\nu=0}^{\left\lfloor\frac{n-j-1}{2}\right\rfloor}\left(\begin{array}{c}
n-j \\
2 \nu+1
\end{array}\right) \mathcal{T}^{n-j-2 \nu-1}(z) R^{\nu}(z),
$$

from which the desired assertions follow.

Proof Theorem 2. To abbreviate notation, let us write $P_{n}(z):=P_{n}\left(z, \sigma_{0}\right)$. Then by Lemma 1, the expression

$$
A_{n N}(z):=\left(\frac{z^{N / 2}}{\sqrt{\Theta(z)}}\right)^{n}\left[\gamma\left(P_{n}^{*}+P_{n}\right)(\Theta(z))-\frac{\sqrt{R(z)}}{W(z)}\left(P_{n}^{*}-P_{n}\right)(\Theta(z))\right]
$$

$\sqrt{\Theta(z)}$ in the sense of (5.7), is a polynomial of degree $\leq n N$. Moreover, we claim that $A_{n N}$ is of exact degree $n N$. Therefore, we have to show that the value $A_{n N}^{*}(0)=$ $\left.z^{n N} \overline{A_{n N}(1 / \bar{z})}\right|_{z=0}$, which is the same as the complex conjugation of the coefficient of $z^{n N}$ in $A_{n N}$, is unequal from zero. Note that

$$
\overline{\Theta\left(\frac{1}{\bar{z}}\right)}=\Theta(z), \quad z^{n N} \overline{\left[\frac{1 / \bar{z}^{N / 2}}{\sqrt{\Theta(1 / \bar{z})}}\right]}=\left(\frac{z^{N / 2}}{\sqrt{\Theta(z)}}\right)^{n}, \overline{\left[\frac{\sqrt{R(1 / \bar{z})}}{W(1 / \bar{z})}\right]}=-\frac{\sqrt{R(z)}}{W(z)},
$$

and that $P_{n}, P_{n}^{*}$ have real coefficients (because $\sigma_{0}$ is a symmetric measure). Thus, the polynomial $A_{n N}^{*}$ is of the form

$$
A_{n N}^{*}(z)=\left(\frac{z^{N / 2}}{\sqrt{\Theta(z)}}\right)^{n}\left[\bar{\gamma}\left(P_{n}^{*}+P_{n}\right)(\Theta(z))+\frac{\sqrt{R(z)}}{W(z)}\left(P_{n}^{*}-P_{n}\right)(\Theta(z))\right],
$$

which yields by Proposition 1 and (1.1) that

$$
\overline{A_{n N}^{*}(0)}=\left(\frac{2 \alpha}{L}\right)^{n}\left[\gamma\left(1-a_{n-1}\left(\sigma_{0}\right)\right)+\gamma\left(1+a_{n-1}\left(\sigma_{0}\right)\right)\right]=2 \gamma\left(\frac{2 \alpha}{L}\right)^{n} \neq 0
$$

Hence,

$$
P_{n N}(z, \sigma):=\frac{1}{2 \gamma}\left(\frac{L}{2 \alpha}\right)^{n} A_{n N}(z)=z^{n N}+\cdots
$$

is a monic polynomial of degree $n N$.

In an analogous way, one can show that $\Omega_{n N}(z, \sigma)$, given as in the theorem, is also a polynomial of degree $n N$ with leading coefficient $F(0, \sigma)=\operatorname{Re} \gamma \neq 0$.

Next, we will apply Theorem 1 to show the desired orthogonality property of $P_{n N}(z, \sigma)$, i.e., we have to verify

$$
\begin{aligned}
& P_{n N}(z, \sigma) F(z, \sigma)+\Omega_{n N}(z, \sigma)=\mathcal{O}\left(z^{n N}\right), \\
& P_{n N}^{*}(z, \sigma) F(z, \sigma)-\Omega_{n N}^{*}(z, \sigma)=\mathcal{O}\left(z^{n N+1}\right), \quad \text { as } \quad z \rightarrow 0 .
\end{aligned}
$$


In a first step, let us denote as above

$$
\begin{aligned}
A_{n N}(z) & :=\left(\frac{z^{N / 2}}{\sqrt{\Theta(z)}}\right)^{n}\left[\gamma\left(P_{n}+P_{n}^{*}\right)(\Theta(z))+\frac{\sqrt{R(z)}}{W(z)}\left(P_{n}-P_{n}^{*}\right)(\Theta(z))\right] \\
B_{n N}(z) & :=\left(\frac{z^{N / 2}}{\sqrt{\Theta(z)}}\right)^{n}\left[\left(\Omega_{n}+\Omega_{n}^{*}\right)(\Theta(z))+\gamma \frac{W(z)}{\sqrt{R(z)}}\left(\Omega_{n}-\Omega_{n}^{*}\right)(\Theta(z))\right] \\
\mathcal{G}(z) & :=\frac{W(z)}{\sqrt{R(z)}} F\left(\Theta(z), \sigma_{0}\right)
\end{aligned}
$$

where, again for abbreviation, $P_{n}:=P_{n}\left(\cdot, \sigma_{0}\right)$ and $\Omega_{n}:=\Omega_{n}\left(\cdot, \sigma_{0}\right)$. Then we have

$$
\begin{aligned}
\left(\frac{\sqrt{\Theta(z)}}{z^{N / 2}}\right)^{n}\left[A_{n N}(z) \mathcal{G}(z)+B_{n N}(z)\right] \\
=P_{n}(\Theta(z))\left(\gamma+\frac{\sqrt{R(z)}}{W(z)}\right) \frac{W(z)}{\sqrt{R(z)}} F\left(\Theta(z), \sigma_{0}\right)+\Omega_{n}(\Theta(z))\left(1+\gamma \frac{W(z)}{\sqrt{R(z)}}\right) \\
\quad+P_{n}^{*}(\Theta(z))\left(\gamma-\frac{\sqrt{R(z)}}{W(z)}\right) \frac{W(z)}{\sqrt{R(z)}} F\left(\Theta(z), \sigma_{0}\right)+\Omega_{n}^{*}(\Theta(z))\left(1-\gamma \frac{W(z)}{\sqrt{R(z)}}\right) \\
=\left(1+\gamma \frac{W(z)}{\sqrt{R(z)}}\right)\left(P_{n}(\Theta(z)) F\left(\Theta(z), \sigma_{0}\right)+\Omega_{n}(\Theta(z))\right) \\
\quad-\left(1-\gamma \frac{W(z)}{\sqrt{R(z)}}\right)\left(P_{n}^{*}(\Theta(z)) F\left(\Theta(z), \sigma_{0}\right)-\Omega_{n}^{*}(\Theta(z))\right) \\
=\mathcal{O}\left(z^{n N}\right)+\mathcal{O}\left(z^{(n+1) N}\right)=\mathcal{O}\left(z^{n N}\right) \quad \text { as } z \rightarrow 0 .
\end{aligned}
$$

For the last identity, we made use of $\Theta(z)=\mathcal{O}\left(z^{N}\right)$ as $z \rightarrow 0$ and of the fact that $P_{n}$ is orthogonal with respect to $F\left(z, \sigma_{0}\right)$; compare Theorem 1 .

In an analogous way, one gets

$$
\begin{aligned}
\left(\frac{\sqrt{\Theta(z)}}{z^{N / 2}}\right)^{n}\left[A_{n N}^{*}(z) \mathcal{G}(z)-B_{n N}^{*}(z)\right] \\
=\left(\bar{\gamma} \frac{W(z)}{\sqrt{R(z)}}-1\right)\left(P_{n}(\Theta(z)) F\left(\Theta(z), \sigma_{0}\right)+\Omega_{n}(\Theta(z))\right) \\
\quad+\left(1+\bar{\gamma} \frac{W(z)}{\sqrt{R(z)}}\right)\left(P_{n}^{*}(\Theta(z)) F\left(\Theta(z), \sigma_{0}\right)-\Omega_{n}^{*}(\Theta(z))\right) \\
=\mathcal{O}(z) \mathcal{O}\left(z^{n N}\right)+\mathcal{O}\left(z^{(n+1) N}\right)=\mathcal{O}\left(z^{n N+1}\right) \quad \text { as } z \rightarrow 0 .
\end{aligned}
$$

Note that $\bar{\gamma} W(z) / \sqrt{R(z)}$ is analytic at $z=0$ and $\bar{\gamma} W(0) / \sqrt{R(0)}=1$, hence we have $\bar{\gamma} W(z) / \sqrt{R(z)}-1=\mathcal{O}(z)$. Summing up, according to $\Theta(z) / z^{N}=\mathcal{O}(1)$, we have shown that

$$
\begin{aligned}
A_{n N}(z) \mathcal{G}(z)+B_{n N}(z) & =\mathcal{O}\left(z^{n N}\right), \\
A_{n N}^{*}(z) \mathcal{G}(z)-B_{n N}^{*}(z) & =\mathcal{O}\left(z^{n N+1}\right), \quad \text { as } z \rightarrow 0
\end{aligned}
$$


Now recall that

$$
\begin{aligned}
P_{n N}(z, \sigma) & =\frac{1}{2 \gamma}\left(\frac{L}{2 \alpha}\right)^{n} A_{n N}(z) \\
\Omega_{n N}(z, \sigma) & =\frac{1}{2 \gamma}\left(\frac{L}{2 \alpha}\right)^{n}\left(B_{n N}(z)+i \operatorname{Im} \gamma A_{n N}(z)\right) \\
F(z, \sigma) & =\mathcal{G}(z)-i \operatorname{Im} \gamma
\end{aligned}
$$

By (5.11),

$$
\begin{aligned}
& P_{n N}(z, \sigma) F(z, \sigma)+\Omega_{n N}(z, \sigma)=\frac{1}{2 \gamma}\left(\frac{L}{2 \alpha}\right)^{n}\left(A_{n N}(z) \mathcal{G}(z)+B_{n N}(z)\right)=\mathcal{O}\left(z^{n N}\right) \\
& P_{n N}^{*}(z, \sigma) F(z, \sigma)-\Omega_{n N}^{*}(z, \sigma)=\frac{1}{2 \gamma}\left(\frac{L}{2 \alpha}\right)^{n}\left(A_{n N}^{*}(z) \mathcal{G}(z)-B_{n N}^{*}(z)\right)=\mathcal{O}\left(z^{n N+1}\right),
\end{aligned}
$$

which is (5.8).

For the proofs of Corollary 1, Theorem 3, Corollary 2, and Theorem 4, we will need Corollary 3 and Theorem 5 . Hence, we prove them first.

Proof of Corollary 3. By (1.4), the polynomials $P_{n N}(z, \sigma)$ and $\Omega_{n N}(z, \sigma)$ satisfy a relation of the form

$$
P_{n N}^{*}(z, \sigma) \Omega_{n N}(z, \sigma)+P_{n N}(z, \sigma) \Omega_{n N}(z, \sigma)=2 \operatorname{Re} \gamma d_{n N}(\sigma) z^{n N}
$$

where $d_{n N}(\sigma):=\prod_{j=0}^{n N-1}\left(1-\left|a_{j}(\sigma)\right|^{2}\right)$. Let the polynomials $A_{n N}$ and $B_{n N}$ be defined as in (5.9) and (5.10), respectively. Then we have

$$
\begin{aligned}
& \frac{4^{n+1}}{L^{2 n}} P_{n N}^{*}(z, \sigma) \Omega_{n N}(z, \sigma)=A_{n N}^{*}(z) B_{n N}(z)+i \operatorname{Im} \gamma A_{n N}^{*}(z) A_{n N}(z), \\
& \frac{4^{n+1}}{L^{2 n}} P_{n N}(z, \sigma) \Omega_{n N}^{*}(z, \sigma)=A_{n N}(z) B_{n N}^{*}(z)-i \operatorname{Im} \gamma A_{n N}(z) A_{n N}^{*}(z),
\end{aligned}
$$

i.e., by straightforward calculation (again $P_{n}:=P_{n}\left(\cdot, \sigma_{0}\right), \Omega_{n}:=\Omega_{n}\left(\cdot, \sigma_{0}\right)$ )

$$
\begin{aligned}
\frac{4^{n+1}}{L^{2 n}}\left(P_{n N}^{*}\right. & \left.(z, \sigma) \Omega_{n N}(z, \sigma)+P_{n N}(z, \sigma) \Omega_{n N}^{*}(z, \sigma)\right) \\
& =A_{n N}^{*}(z) B_{n N}(z)+A_{n N}(z) B_{n N}^{*}(z) \\
& =2(\bar{\gamma}+\gamma)\left(\frac{z^{N / 2}}{\sqrt{\Theta(z)}}\right)^{2 n}\left(P_{n}^{*} \Omega_{n}+P_{n} \Omega_{n}^{*}\right)(\Theta(z)) \\
& =8 \operatorname{Re} \gamma d_{n}\left(\sigma_{0}\right)\left(\frac{z^{N / 2}}{\sqrt{\Theta(z)}}\right)^{2 n} \Theta^{n}(z) \quad(\text { by }(1.4)) \\
& =8 \operatorname{Re} \gamma d_{n}\left(\sigma_{0}\right) z^{n N} .
\end{aligned}
$$

Comparing this identity with (5.12) and taking into consideration that by (5.2), $\operatorname{Re} \gamma>0$, we obtain

$$
d_{n N}(\sigma)=\left(\frac{L}{2}\right)^{2 n} d_{n}\left(\sigma_{0}\right)
$$

and the assertion follows by an induction argument. 
Proof of Theorem 5. From (4.2) we obtain (cf. [16, Thm.3.1]),

$$
\begin{aligned}
P_{N}^{(n N)}(z, \sigma)=\frac{1}{2 d_{n N}(\sigma) z^{n N}}\left[P_{(n+1) N}(\right. & z, \sigma)\left(\hat{\Omega}_{n N}(z, \sigma)+\hat{\Omega}_{n N}^{*}(z, \sigma)\right) \\
& \left.-\hat{\Omega}_{(n+1) N}(z, \sigma)\left(P_{n N}(z, \sigma)-P_{n N}^{*}(z, \sigma)\right)\right] .
\end{aligned}
$$

Let the polynomials $A_{n N}, B_{n N}$ and $A_{(n+1) N}, B_{(n+1) N}$ be defined as in (5.9) and (5.10), respectively, and then we have, by Theorem 2 ,

$$
\begin{aligned}
P_{N}^{(n N)}(z, \sigma)= & \frac{L^{2 n+1}}{2^{2 n+4} d_{n N}(\sigma) \alpha^{n+1} \gamma \operatorname{Re} \gamma z^{n N}}\left[A_{(n+1) N}(z)\left(\bar{\alpha}^{n} \bar{\gamma} B_{n N}(z)+\alpha^{n} \gamma B_{n N}^{*}(z)\right)\right. \\
& \left.-B_{(n+1) N}(z)\left(\bar{\alpha}^{n} \bar{\gamma} A_{n N}(z)-\alpha^{n} \gamma A_{n N}^{*}(z)\right)\right] \\
& +\frac{1}{2 d_{n N}(\sigma) z^{n N}}\left[P_{(n+1) N}(z, \sigma)\left(\frac{i \operatorname{Im} \gamma}{\operatorname{Re} \gamma} P_{n N}(z, \sigma)-\frac{i \operatorname{Im} \gamma}{\operatorname{Re} \gamma} P_{n N}^{*}(z, \sigma)\right)\right. \\
& -\frac{i \operatorname{Im} \gamma}{\operatorname{Re} \gamma} P_{(n+1) N}(z, \sigma)\left(P_{n N}(z, \sigma)-P_{n N}^{*}(z, \sigma)\right] .
\end{aligned}
$$

The second term in the sum on the right-hand side vanishes and a tedious but straightforward calculation gives

$$
\begin{aligned}
& P_{N}^{(n N)}(z, \sigma)=\frac{K z^{N / 2}}{[\Theta(z)]^{n+1 / 2}} \times \\
& \quad\left[\operatorname { R e } \{ \alpha ^ { n } \gamma \} \left\{\gamma\left(\left(P_{n+1}+P_{n+1}^{*}\right)\left(\Omega_{n}+\Omega_{n}^{*}\right)-\left(\Omega_{n+1}-\Omega_{n+1}^{*}\right)\left(P_{n}-P_{n}^{*}\right)\right)(\Theta(z))\right.\right. \\
& \left.\quad+\frac{\sqrt{R(z)}}{W(z)}\left(\left(P_{n+1}-P_{n+1}^{*}\right)\left(\Omega_{n}+\Omega_{n}^{*}\right)-\left(\Omega_{n+1}+\Omega_{n+1}^{*}\right)\left(P_{n}-P_{n}^{*}\right)\right)(\Theta(z))\right\} \\
& -i \operatorname{Im}\left\{\alpha^{n}\right\}\left\{\left(\left(P_{n+1}-P_{n+1}^{*}\right)\left(\Omega_{n}-\Omega_{n}^{*}\right)-\left(\Omega_{n+1}+\Omega_{n+1}^{*}\right)\left(P_{n}+P_{n}^{*}\right)\right)(\Theta(z))\right. \\
& \left.\left.\quad+\frac{\gamma W(z)}{\sqrt{R(z)}}\left(\left(P_{n+1}+P_{n+1}^{*}\right)\left(\Omega_{n}-\Omega_{n}^{*}\right)-\left(\Omega_{n+1}-\Omega_{n+1}^{*}\right)\left(P_{n}+P_{n}^{*}\right)\right)(\Theta(z))\right\}\right]
\end{aligned}
$$

where the constant $K$ is given by

$$
K=\frac{L^{2 n+1}}{2^{2 n+3} d_{n N}(\sigma) \alpha^{n+1} \gamma \operatorname{Re} \gamma}
$$

and where $P_{\nu}:=P_{\nu}\left(\cdot, \sigma_{0}\right), \Omega_{\nu}:=\Omega_{\nu}\left(\cdot, \sigma_{0}\right)$ with $\nu \in\{n, n+1\}$. Using the identities $[16$, Cor.3.1],

$$
\begin{aligned}
& 2 P_{n+1}=\left(P_{n}+P_{n}^{*}\right) P_{1}^{(n)}+\left(P_{n}-P_{n}^{*}\right) \Omega_{1}^{(n)}, \\
& 2 \Omega_{n+1}=\left(\Omega_{n}-\Omega_{n}^{*}\right) P_{1}^{(n)}+\left(\Omega_{n}+\Omega_{n}^{*}\right) \Omega_{1}^{(n)},
\end{aligned}
$$

$P_{1}^{(n)}=P_{1}^{(n)}\left(\cdot, \sigma_{0}\right)$ and $\Omega_{1}^{(n)}=\Omega_{1}^{(n)}\left(\cdot, \sigma_{0}\right)$, we get

$$
\begin{aligned}
& \left(P_{n+1} \pm P_{n+1}^{*}\right)\left(\Omega_{n}+\Omega_{n}^{*}\right)-\left(\Omega_{n+1} \mp \Omega_{n+1}^{*}\right)\left(P_{n}-P_{n}^{*}\right)=2 d_{n}\left(\sigma_{0}\right) z^{n}\left(P_{1}^{(n)} \pm P_{1}^{(n) *}\right), \\
& \left(P_{n+1} \pm P_{n+1}^{*}\right)\left(\Omega_{n}-\Omega_{n}^{*}\right)-\left(\Omega_{n+1} \mp \Omega_{n+1}^{*}\right)\left(P_{n}+P_{n}^{*}\right)=-2 d_{n}\left(\sigma_{0}\right) z^{n}\left(\Omega_{1}^{(n)} \mp \Omega_{1}^{(n) *}\right) .
\end{aligned}
$$


Now the above representation simplifies to

$$
\begin{aligned}
P_{N}^{(n N)}(z, \sigma)=\frac{2 K d_{n}\left(\sigma_{0}\right) z^{N / 2}}{\sqrt{\Theta(z)}}\left[\gamma \operatorname{Re}\left\{\alpha^{n} \gamma\right\}\left(P_{1}^{(n)}(\Theta(z))+P_{1}^{(n) *}(\Theta(z))\right)\right. \\
+\operatorname{Re}\left\{\alpha^{n} \gamma\right\} \frac{\sqrt{R(z)}}{W(z)}\left(P_{1}^{(n)}(\Theta(z))-P_{1}^{(n) *}(\Theta(z))\right) \\
+i \operatorname{Im}\left\{\alpha^{n}\right\}\left(\Omega_{1}^{(n)}(\Theta(z))+\Omega_{1}^{(n) *}(\Theta(z))\right) \\
\left.+i \gamma \operatorname{Im}\left\{\alpha^{n}\right\} \frac{W(z)}{\sqrt{R(z)}}\left(\Omega_{1}^{(n)}(\Theta(z))-\Omega_{1}^{(n) *}(\Theta(z))\right)\right]
\end{aligned}
$$

Further, from

$$
\begin{aligned}
& P_{1}^{(n)}(z) \pm P_{1}^{(n) *}(z)=\left(1 \mp a_{n}\left(\sigma_{0}\right)\right)(z \pm 1) \\
& \Omega_{1}^{(n)}(z) \pm \Omega_{1}^{(n) *}(z)=\left(1 \pm a_{n}\left(\sigma_{0}\right)\right)(z \pm 1)
\end{aligned}
$$

and

$$
z^{N / 2} \sqrt{\Theta(z)}=\frac{\mathcal{T}(z)-\sqrt{R(z)}}{L}, \quad \frac{z^{N / 2}}{\sqrt{\Theta(z)}}=\frac{\mathcal{T}(z)+\sqrt{R(z)}}{L},
$$

we get, with the aid of (5.13),

$$
\begin{aligned}
P_{N}^{(n N)}(z, \sigma)= & \frac{1}{2 \alpha^{n+1} \gamma \operatorname{Re} \gamma}\left\{\operatorname{Re}\left\{\alpha^{n} \gamma\right\}\left[\gamma\left(1-a_{n}\left(\sigma_{0}\right)\right) \mathcal{T}(z)-\left(1+a_{n}\left(\sigma_{0}\right)\right) \frac{R(z)}{W(z)}\right]\right. \\
& \left.+i \operatorname{Im}\left\{\alpha^{n}\right\}\left[\left(1+a_{n}\left(\sigma_{0}\right)\right) \mathcal{T}(z)-\gamma\left(1-a_{n}\left(\sigma_{0}\right)\right) W(z)\right]\right\} .
\end{aligned}
$$

This is the desired result.

Now we are ready to prove the remaining results of Section 3.

Proof of Corollary 1. The leading coefficient of an orthonormal polynomial $\Phi_{n}(z, \mu)=$ $k_{n}(\mu) z^{n}+\cdots$ can be expressed in terms of the corresponding reflection coefficients $\left\{a_{n}(\mu)\right\}$ by the formula

$$
k_{n}(\mu)=\left(c_{0}(\mu) \prod_{j=0}^{n-1}\left(1-\left|a_{j}(\mu)\right|^{2}\right)\right)^{-1 / 2}, \quad c_{0}(\mu)=\int_{0}^{2 \pi} d \mu(\varphi)=F(0, \mu),
$$

e.g., $[8,(2.7)$ and (4.2)]. Using Corollary 3 and the fact that $F(0, \sigma)=\operatorname{Re} \gamma$, we obtain from Theorem 2

$$
\begin{aligned}
\sqrt{\operatorname{Re} \gamma} \Phi_{n N}(z, \sigma)=\frac{1}{2 \gamma \alpha^{n}}\left(\frac{z^{N}}{\Theta(z)}\right)^{n / 2}\left\{\Phi_{n}\left(\Theta(z), \sigma_{0}\right)\left(\gamma+\frac{\sqrt{R(z)}}{W(z)}\right)\right. & \\
& \left.+\Phi_{n}^{*}\left(\Theta(z), \sigma_{0}\right)\left(\gamma-\frac{\sqrt{R(z)}}{W(z)}\right)\right\}
\end{aligned}
$$

and the assertion follows by (3.1).

Proof of Theorem 3. (a) Since $\Theta(z)=\mathcal{O}\left(z^{N}\right)$ as $z \rightarrow 0$ and $F\left(0, \sigma_{0}\right)=1$, the first $N$ coefficients $c_{0}(\sigma), \ldots, c_{N-1}(\sigma)$ in the power series expansion,

$$
F(z, \sigma)=\frac{W(z)}{\sqrt{R(z)}} F\left(\Theta(z), \sigma_{0}\right)-i \operatorname{Im} \gamma=: c_{0}(\sigma)+2 \sum_{j=1}^{\infty} c_{j}(\sigma) z^{j}, \quad|z|<1,
$$


are independent of $\sigma_{0}$. But then, by [8, formula (3.2)], the reflection coefficients $a_{0}(\sigma), \ldots, a_{N-2}(\sigma)$ are independent of $\sigma_{0}$ as well.

(b) We first consider the case that $\alpha=1$, i.e., we have to show that

$$
a_{n N+j}(\sigma)=a_{j}(\sigma) \quad \text { for } \quad j=0,1, \ldots, N-2
$$

and

$$
a_{(n+1) N-1}(\sigma)=\frac{a_{n}\left(\sigma_{0}\right)\left(\gamma^{2}+1\right)+\gamma^{2}-1}{2} .
$$

In order to see (5.14), it suffices to prove that all the polynomials, $P_{N-1}^{(n N)}(z, \sigma), n \in \mathbb{N}_{0}$, coincide, cf. [8, Thm.9.2]. Here, the $n N$-th associated polynomials $P_{N-1}^{(n N)}(z, \sigma)$ are given as in (4.1). From the recurrence relation (1.1), one derives that these polynomials are explicitly given by

$$
z P_{N-1}^{(n N)}(z, \sigma)=\frac{P_{N}^{(n N)}(z, \sigma)-P_{N}^{(n N)}(0, \sigma) P_{N}^{(n N) *}(z, \sigma)}{1-\left|P_{N}^{(n N)}(0, \sigma)\right|^{2}} .
$$

Furthermore, by Theorem 5, the polynomials $P_{N}^{(n N)}$ are of the form

$$
P_{N}^{(n N)}(z, \sigma)=\beta_{n} \mathcal{T}(z)+\lambda_{n} \frac{R(z)}{W(z)}
$$

where

$$
\beta_{n}=\frac{1-a_{n}\left(\sigma_{0}\right)}{2} \text { and } \lambda_{n}=-\frac{1+a_{n}\left(\sigma_{0}\right)}{2 \gamma} .
$$

In order to avoid excessive notation, let us abbreviate

$$
c_{n}:=-\overline{a_{(n+1) N-1}}=P_{N}^{(n N)}(0, \sigma)=\beta_{n}+\frac{\lambda_{n}}{\gamma} .
$$

Then the identity $P_{N-1}^{(n N)}(z, \sigma) \equiv P_{N-1}^{((n+1) N)}(z, \sigma)$ holds if and only if

$$
\begin{aligned}
\left(1-\left|c_{n+1}\right|^{2}\right)\left[P_{N}^{(n N)}(z, \sigma)\right. & \left.-c_{n} P_{N}^{(n N) *}(z, \sigma)\right] \\
& =\left(1-\left|c_{n}\right|^{2}\right)\left[P_{N}^{((n+1) N)}(z, \sigma)-c_{n+1} P_{N}^{((n+1) N) *}(z, \sigma)\right] .
\end{aligned}
$$

Taking into consideration that

$$
P_{N}^{(n N) *}(z, \sigma)=\beta_{n} \mathcal{T}(z)-\bar{\lambda}_{n} \frac{R(z)}{W(z)},
$$

the above equality also can be written as

$$
\begin{aligned}
\left(1-\left|c_{n+1}\right|^{2}\right)( & \left.\beta_{n}\left(1-c_{n}\right) \mathcal{T}(z)+\left(\lambda_{n}+c_{n} \bar{\lambda}_{n}\right) \frac{R(z)}{W(z)}\right) \\
= & \left(1-\left|c_{n}\right|^{2}\right)\left(\beta_{n+1}\left(1-c_{n+1}\right) \mathcal{T}(z)+\left(\lambda_{n+1}+c_{n+1} \bar{\lambda}_{n+1}\right) \frac{R(z)}{W(z)}\right) .
\end{aligned}
$$

Hence, for all $n \in \mathbb{N}_{0}$, the following relations remain to be shown :

$$
\begin{aligned}
\beta_{n}\left(1-\left|c_{n+1}\right|^{2}\right)\left(1-c_{n}\right) & =\beta_{n+1}\left(1-\left|c_{n}\right|^{2}\right)\left(1-c_{n+1}\right), \\
\left(1-\left|c_{n+1}\right|^{2}\right)\left(\lambda_{n}+c_{n} \bar{\lambda}_{n}\right) & =\left(1-\left|c_{n}\right|^{2}\right)\left(\lambda_{n+1}+c_{n+1} \bar{\lambda}_{n+1}\right) .
\end{aligned}
$$


Substituting the corresponding expressions from (5.17) and (5.18), the first identity in (5.19) turns out to be

$$
\begin{aligned}
\left(1-a_{n+1}^{2}\left(\sigma_{0}\right)\right)\left(4-\left|\gamma\left(1-a_{n}\left(\sigma_{0}\right)\right)-\frac{1+a_{n}\left(\sigma_{0}\right)}{\gamma}\right|^{2}\right) \\
=\left(1-a_{n}^{2}\left(\sigma_{0}\right)\right)\left(4-\left|\gamma\left(1-a_{n+1}\left(\sigma_{0}\right)\right)-\frac{1+a_{n+1}\left(\sigma_{0}\right)}{\gamma}\right|^{2}\right) .
\end{aligned}
$$

Now note that the function

$$
h(x, y):=\left(1-x^{2}\right)\left(4-\left|\gamma(1-y)-\frac{1+y}{\gamma}\right|^{2}\right), \quad|\gamma|=1,
$$

from $\mathbb{R}^{2}$ to $\mathbb{R}$ is symmetric in the sense that $h(x, y)=h(y, x)$. This can be seen easily by writing

$$
\begin{aligned}
h(x, y) & =\left(1-x^{2}\right)\left(4-\left[\gamma(1-y)-\frac{1+y}{\gamma}\right]\left[\frac{1-y}{\gamma}-\gamma(1+y)\right]\right) \\
& =\left(1-x^{2}\right)\left(4-\left[2\left(1+y^{2}\right)-\left(1-y^{2}\right)\left(\gamma^{2}+\frac{1}{\gamma^{2}}\right)\right]\right) \\
& =\left(1-x^{2}\right)\left(1-y^{2}\right)\left(2+\gamma^{2}+\frac{1}{\gamma^{2}}\right) .
\end{aligned}
$$

The setting $x=a_{n+1}\left(\sigma_{0}\right)$ and $y=a_{n}\left(\sigma_{0}\right)$ gives the first identity in (5.19). The second one can be shown in a similar way. This proves (5.14).

The desired representation (5.15) of the reflection coefficients, $a_{(n+1) N-1}(\sigma), n \in$ $\mathbb{N}_{0}$, follows from

$$
\begin{aligned}
a_{(n+1) N-1}(\sigma) & =-\overline{P_{N}^{(n N)}(0, \sigma)}=-\left(\beta_{n}+\gamma \bar{\lambda}_{n}\right) \\
& =\frac{1}{2}\left[\left(a_{n}\left(\sigma_{0}\right)-1\right)+\gamma^{2}\left(a_{n}\left(\sigma_{0}\right)+1\right)\right] \\
& =\frac{a_{n}\left(\sigma_{0}\right)\left(1+\gamma^{2}\right)+\gamma^{2}-1}{2} .
\end{aligned}
$$

Let us now consider the general situation that $\mathcal{T}(z)=\alpha z^{N}+\cdots,|\alpha|=1$. Then the polynomials

$$
\hat{\mathcal{T}}(z):=d^{N / 2} \mathcal{T}\left(\frac{z}{d}\right)=z^{N}+\cdots \quad \text { and } \quad \hat{R}(z)=d^{N} R\left(\frac{z}{d}\right)=z^{2 N}+\cdots
$$

where $d:=\alpha^{2 / N}$, are monic polynomials and the transformation

$$
\hat{\Theta}(z)=\frac{\hat{\mathcal{T}}(z)-\sqrt{\hat{R}(z)}}{\hat{\mathcal{T}}(z)+\sqrt{\hat{R}(z)}}
$$

is of the form (2.6). Now the mapping $\hat{\Theta}$ acts on the set

$$
\hat{E}_{N}:=\prod_{j=1}^{N}\left[\hat{\varphi}_{2 j-1}, \hat{\varphi}_{2 j}\right]
$$

where $\hat{\varphi}_{\nu}=\varphi_{\nu}+\arg d$. This means that the $\operatorname{arcs} \Gamma_{\hat{E}_{N}}$, generated by the polynomials $\hat{R}$ and $\hat{\mathcal{T}}$, result from the $\operatorname{arcs} \Gamma_{E_{N}}$ by a simple rotation of the angle $\arg d$. 
Further, it is not difficult to show that the polynomials $P_{n N}(z, \sigma)$ and

$$
\begin{aligned}
\hat{P}_{n N}(z, \hat{\sigma}):=\hat{\kappa}_{n}\left(\frac{z^{N / 2}}{\sqrt{\hat{\Theta}(z)}}\right)^{n}\left\{P_{n}\left(\hat{\Theta}(z), \sigma_{0}\right)(\gamma\right. & \left.\frac{\sqrt{\hat{R}(z)}}{\hat{W}(z)}\right) \\
& \left.+P_{n}^{*}\left(\hat{\Theta}(z), \sigma_{0}\right)\left(\gamma-\frac{\sqrt{\hat{R}(z)}}{\hat{W}(z)}\right)\right\}
\end{aligned}
$$

where $\hat{W}(z):=d^{N / 2} W(z / d)$ and $\hat{\kappa}_{n}=L^{n} / 2^{n+1} \gamma$ are related by

$$
\hat{P}_{n N}(z, \hat{\sigma})=d^{n N} P_{n N}\left(\frac{z}{d}\right) .
$$

Here, the measure $\hat{\sigma}$ is given as in (2.11) by using the transformation $\hat{\Theta}$ instead of $\Theta$. Hence, as pointed out in [19, Remark 4.2], the reflection coefficients $\left\{a_{n}(\hat{\sigma})=\right.$ $\left.-\overline{P_{n+1}(0, \hat{\sigma})}\right\}$ can be expressed in terms of $\left\{a_{n}(\sigma)\right\}$ by

$$
a_{n}(\hat{\sigma})=d^{-(n+1)} a_{n}(\sigma), \quad n \in \mathbb{N} .
$$

By the proved above part for $\alpha=1$, the reflection coefficients $a_{n}(\hat{\sigma})$ satisfy (5.14) and (5.15). Together with (5.20), this proves the theorem.

Proof of Corollary 2. By Theorem 3, the reflection coefficients $a_{0}(\sigma), \ldots, a_{N-2}(\sigma)$ do not depend on the measure $\sigma_{0}$. Hence, if we are interested only in the first $N-1$ reflection coefficients of $\sigma$, we can consider the simplest case $F\left(z, \sigma_{0}\right) \equiv 1$, i.e., $d \sigma_{0}(\varphi)=d \varphi$, which gives

$$
F(z, \sigma)=\frac{W(z)}{\sqrt{R(z)}}=\sqrt{\frac{W(z)}{V(z)}}
$$

(recall that $\gamma=1$ ) where $R=V W$. The assertion follows from [22, Ex.6.5, pp. 494496].

Proof of Theorem 4 . We first show that the polynomials $R, \mathcal{T}$, and $W$ from the theorem fulfill the assumptions from Section 2. By [12, Thm.6.2], all the zeros of the polynomials

$$
z P_{N-1}(z, \sigma) \pm P_{N-1}^{*}(z, \sigma) \text { and } z \hat{\Omega}_{N-1}(z, \sigma)-\hat{\Omega}_{N-1}^{*}(z, \sigma)
$$

are simple and located on the unit circle. Furthermore, the zeros of $z P_{N-1}(z, \sigma)-$ $P_{N-1}^{*}(z, \sigma)$ and $z \hat{\Omega}_{N-1}(z, \sigma)-\hat{\Omega}_{N-1}^{*}(z, \sigma)$ separate each other as it follows from [22, Beispiel 6.6,p.496]. Finally, by [22, Lemma 6.13, p.489], we have

$$
z P_{N-1}(z, \sigma)+P_{N-1}^{*}(z, \sigma)=z \hat{\Omega}_{N-1}(z, \sigma)+\hat{\Omega}_{N-1}^{*}(z, \sigma) .
$$

Using this identity and relation (1.4), it is not difficult to see that

$$
\mathcal{T}^{2}(z)-R(z)=4 d_{N}(\sigma) .
$$

All these facts together show that our theory works with the triple $(R, \mathcal{T}, W)$.

In order to prove the theorem, it suffices to show that the C-functions $F(z, \sigma)$ and $F\left(z, \sigma_{0}\right)$ are related to each other by

$$
F(z, \sigma)=\frac{W(z)}{\sqrt{R(z)}} F\left(\Theta(z), \sigma_{0}\right),
$$


since $\gamma=W(0) / \sqrt{R(0)}=1$. Or, to use different words, we have to demonstrate that the reflection coefficients corresponding to the C-function at the right-hand side in (5.21) satisfy (1)-(4). By Proposition 2, the right-hand side in (5.21) indeed gives a C-function, which we will denote now by $\mathcal{G}$ and its associated reflection coefficients by $\left\{b_{n}\right\}$. Let us show that $b_{n}=a_{n}(\sigma)$. Since $b_{0}, \ldots, b_{N-2}$ are independent of $\sigma_{0}$, recall Theorem 3 , we may choose $F\left(z, \sigma_{0}\right) \equiv 1$. Then the first $N-1$ identities

$$
b_{n}=a_{n}(\sigma), \quad n=0, \ldots, N-2,
$$

can be derived from [22, Beispiel 6.5 and 6.6, p.494]. Moreover, Theorem 3 gives the properties (3) and (4), while (1) is obvious. Summing up, we have shown that $\left\{b_{n}\right\}=\left\{a_{n}(\sigma)\right\}$ which proves (5.21).

Acknowledgment. The authors would like to thank the referees for some valuable comments. This work was supported by the Austrian Fonds zur Förderung der wissenschaftlichen Forschung, project-number P10737-TEC.

\section{References}

1. V. M. Badkov, Systems of orthogonal polynomials explicitly represented by the Jacobi polynomials, Math. Notes 42 (1987), 858-863.

2. D. Barrios and G. López, Ratio asymptotics of polynomials on arcs of the unit circle, to appear.

3. M. Bello and G. López, Ratio and relative asymptotics of polynomials on an arc of the unit circle, to appear.

4. D. Bessis and P. Moussa, Orthogonality properties of iterated polynomial mappings, Comm. Math. Phys. 88 (1983), 503-529.

5. P. L. Duren, Theory of $H^{p}$ spaces, Academic Press, New York, 1970.

6. G. Freud, Orthogonal Polynomials, Akademiai Kiadó and Pergamon Press, New York, 1971.

7. Ya. L. Geronimus, On polynomials orthogonal on a circle, on the trigonometric moment problem, and on the associated functions of Carathéodory's and Schur's type, Mat. Sbornik (N.S.) 15 (1944), 99-130. (in Russian)

8. - Polynomials orthogonal on a circle and their applications. In: Series and Approximations, A.M.S. Transl. Vol.3, Providence, R.I., 1962, pp.1-78.

9. J. S. Geronimo and W. Van Assche, Orthogonal polynomials on several intervals via a polynomial mapping, Trans. A.M.S. 308 (1988), 559-581.

10. L. Golinskii, P. Nevai, F. Pinter, and W. Van Assche, Perturbation of orthogonal polynomials on an arc of the unit circle $I I$, to appear.

11. M. E. Ismail and X. Li, On sieved orthogonal polynomials IX: Orthogonality on the unit circle, Pacific J. Math. 153 (1992), 289-297.

12. W. B. Jones, O. Njåstad and W. J. Thron, Moment theory, orthogonal polynomials, quadrature, and continued fractions associated with the unit circle, Bull. London Math. Soc. 21 (1989), 113-152.

13. P. Koosis, Introduction to $H_{p}$ Spaces, London Mathematical Society, Lecture Note Series, Vol.40, Cambridge University Press, 1980.

14. F. Marcellán and G. Sansigre, Orthogonal polynomials on the unit circle: Symmetrization and quadratic decomposition, J. Approx. Theory 65 (1991), 109-119.

15. F. Peherstorfer, Linear combinations of orthogonal polynomials generating positive quadrature formulas, Math. Comput. 55 (1990), 231-241.

16. polynomials, Constr. Approx. 12 (1996), 161-186.

17. F. Peherstorfer and R. Steinbauer, Characterization of orthogonal polynomials with respect to a functional, J. Comput. Appl. Math. 65 (1995), 339-355.

18. 140-184.

19. - Orthogonal polynomials on several arcs of the unit circle II. Orthogonal polynomials with periodic reflection coefficients, J. Approx. Theory 87 (1996), 60-102. 
20. - Asymptotic behaviour of orthogonal polynomials on the unit circle with asymptotically periodic reflection coefficients, J. Approx. Theory 88 (1997), 316-353.

21. J. Schur, Über Potenzreihen, die im Inneren des Einheitskreises beschränkt sind, J. Reine Angew. Math. 148 (1918), 122-145. (in German)

22. R. Steinbauer, Orthogonalpolynome auf mehreren Bögen des komplexen Einheitskreises, Ph.D. dissertation, Universitätsverlag Rudolf Trauner, Linz 1995. (in German)

23. G. Szegö, Orthogonal polynomials, 4th ed., A.M.S. Colloq. Publ., Vol.23, A.M.S., Providence, R.I., 1975.

24. L. V. Toralballa, Theory of Functions, Charles E. Merrill Books, Inc., Columbus, Ohio, 1963.

INSTITUT FÜr MAThEMATIK, JOHANNES KEPLER UNIVERSITÄT LiNZ, 4040 LINZ-AUHOF, AUSTRIA

E-mail: franz.peherstorfer@jk.uni-linz.ac.at, robert.steinbauer $\Theta j k$.uni-linz.ac.at 